\title{
Nanotheranostics
}

2022; 6(1): 10-30. doi: 10.7150/ntno.61244

Review

\section{Design and synthesis of gold nanostars-based SERS nanotags for bioimaging applications}

\author{
Bohdan Andreiuk ${ }^{1,2}{ }^{\bowtie}$, Fay Nicolson ${ }^{1,3}$, Louise M. Clark ${ }^{1,2}$, Sajanlal R. Panikkanvalappil1 ${ }^{1}$ Kenry ${ }^{1,4}$, \\ Mohammad Rashidian ${ }^{2}$, Stefan Harmsen ${ }^{-凶}$ and Moritz F. Kircher ${ }^{1,6, \dagger}$ \\ 1. Department of Imaging, Dana-Farber Cancer Institute and Harvard Medical School, Boston, MA 02215, USA. \\ 2. Department of Cancer Immunology and Virology, Dana-Farber Cancer Institute and Harvard Medical School, Boston, MA 02215, USA. \\ 3. Department of Cancer Biology, Dana-Farber Cancer Institute and Harvard Medical School, Boston, MA 02215, USA. \\ 4. Harvard John A. Paulson School of Engineering and Applied Sciences, Harvard University, Cambridge, MA 02138, USA. \\ 5. Department of Radiology, Perelman School of Medicine, University of Pennsylvania, Philadelphia, PA 19104, USA. \\ 6. Department of Radiology, Brigham \& Women's Hospital and Harvard Medical School, Boston, MA 022115, USA. \\ tDeceased August 15, 2020 \\ $\bowtie$ Corresponding authors: Bohdan Andreiuk: bohdan_andreiuk@dfci.harvard.edu. Stefan Harmsen: stefan.harmsen@pennmedicine.upenn.edu \\ (C) The author(s). This is an open access article distributed under the terms of the Creative Commons Attribution License (https://creativecommons.org/licenses/by/4.0/). \\ See http://ivyspring.com/terms for full terms and conditions.
}

Received: 2021.04.04; Accepted: 2021.05.10; Published: 2022.01.01

\begin{abstract}
Surface-enhanced Raman spectroscopy (SERS) nanotags hold a unique place among bioimaging contrast agents due to their fingerprint-like spectra, which provide one of the highest degrees of detection specificity. However, in order to achieve a sufficiently high signal intensity, targeting capabilities, and biocompatibility, all components of nanotags must be rationally designed and tailored to a specific application. Design parameters include fine-tuning the properties of the plasmonic core as well as optimizing the choice of Raman reporter molecule, surface coating, and targeting moieties for the intended application. This review introduces readers to the principles of SERS nanotag design and discusses both established and emerging protocols of their synthesis, with a specific focus on the construction of SERS nanotags in the context of bioimaging and theranostics.
\end{abstract}

Key words: Surface-enhanced Raman scattering, gold nanostar synthesis, near-infrared dye, cancer imaging

\section{Introduction}

First reported in 1928, Raman spectroscopy is the phenomenon of inelastic scattering of light at longer or shorter wavelengths than that of the incident source $[1,2]$. Because such changes in frequency are specific to a particular vibration within the molecule of interest, the composition of the sample can be determined in a non-destructive manner using Raman spectroscopy [3]. However, since only one in $\sim 10^{7}$ photons scatters inelastically, Raman spectroscopy is an inherently weak technology. Interestingly, the Raman cross-section of a molecule can be significantly increased by adsorbing the molecule on a noble metal nanoparticle surface in a process known as surface-enhanced Raman scattering (SERS), which provides a means of overcoming the inherently weak nature of spontaneous Raman scattering.
SERS was first reported in 1974 by Fleischmann et al., who observed an enhancement in the Raman scattering of pyridine adsorbed onto a chemically roughened silver electrode [4]. SERS enhancement is attributed to two factors: electromagnetic enhancement and charge transfer. The former mechanism is dependent on an interaction between the localized surface plasmon resonance (LSPR) of the metallic nanoparticle surface and the adsorbed molecule [3]. When incident laser light interacts with the metallic nanoparticles (NPs), it induces an oscillation of conduction band electrons, which increases polarizability of the adsorbed molecule leading to significant enhancement of Raman scattering [5]. Enhancement factors of $10^{7}-10^{9}$ have been reported [6]. 
Even greater enhancement in Raman signal can be achieved by placing molecules in so-called "hot spots" between NPs, where the electromagnetic field is maximized due to interaction of the localized surface plasmon resonances of neighboring NPs. Additionally, by adsorbing a molecule that has an absorption maximum that is resonant with the incident laser wavelength, additional enhancement is achieved in a process known as "surface-enhanced resonance Raman scattering" (SERRS) [7, 8]. The observed SE(R)RS signal amplification is dependent on a number of factors including the interaction between adjacent NPs and their LSPRs, the number of analyte molecules on the surface of the metallic nanoparticle, and resonance contribution of both the analyte and metal. The resonance contribution of the metal is governed by the type, size, and shape of metallic nanoparticles [9, 10]. For biomedical applications, SERS nanoparticles (or SERS nanotags) typically consist of a plasmonic nanoparticle core coated with molecules (Raman reporters) that are encapsulated in a protective shell that can be decorated with targeting ligands (Figure 1a). Raman reporters are selected not only based on their polarizability and resonance properties but also their binding affinity to the metal NP surface, which increases the overall number of reporters per particle, therefore generating higher signal intensity $[3,11,12]$. The metal/molecule system is encapsulated in a biocompatible coating that stabilizes the metal nanoparticle and prevents desorption of the Raman reporter molecules from the NP's surface [13]. Finally, the resulting $S E(R) R S$ nanotags can be functionalized with targeting ligands that direct the nanoparticles towards specific disease markers to enable their detection using Raman imaging $[12,13]$.
While many have discussed the general principles and applications of SERS, here we will specifically discuss the important aspects of design and synthesis of SE(R)RS nanotags for biomedical applications, focusing on those with star-shaped plasmonic cores - nanostars [10, 11, 14, 15].

\section{Synthesis Strategies for Gold Nanostars}

For biomedical applications, gold and silver nanoparticles are preferred, because their LSPRs are within the visible and near-infrared (NIR) regions of the light spectrum $[11,18]$. Although silver NPs exhibit stronger enhancement, their LSPR is blue-shifted relative to gold nanoparticles (AuNPs). Furthermore, due to their higher rates of biodegradation and release of toxic $\mathrm{Ag}^{+}$ions, silver NPs are associated with poorer biocompatibility [19, 20]. Since AuNPs are inert, biocompatible and their LSPRs can be shifted towards the NIR window - an optical window (700-950 nm) where tissue absorption and autofluorescence are minimized - AuNPs are regarded ideal for in vivo imaging applications [11]. When carrying out in vivo imaging, a laser wavelength in the NIR region (e.g. 785 or $830 \mathrm{~nm}$ ) is typically selected to ensure maximum tissue penetration [21]. Through changes in their size and shape, the LSPR of the AuNPs can be tuned to be in resonance with the excitation laser wavelength [17]. While spherical cores with a diameter of 10-100 nm have an LSPR of 530$580 \mathrm{~nm}$, respectively, the LSPR of anisotropic AuNPs (e.g. rods, cubes, cages, or stars) are red-shifted into the NIR [13, 22-24]. Among these core shapes, gold nanostars are known to generate the highest Raman enhancement factors (Figure 1b) [16, 25].
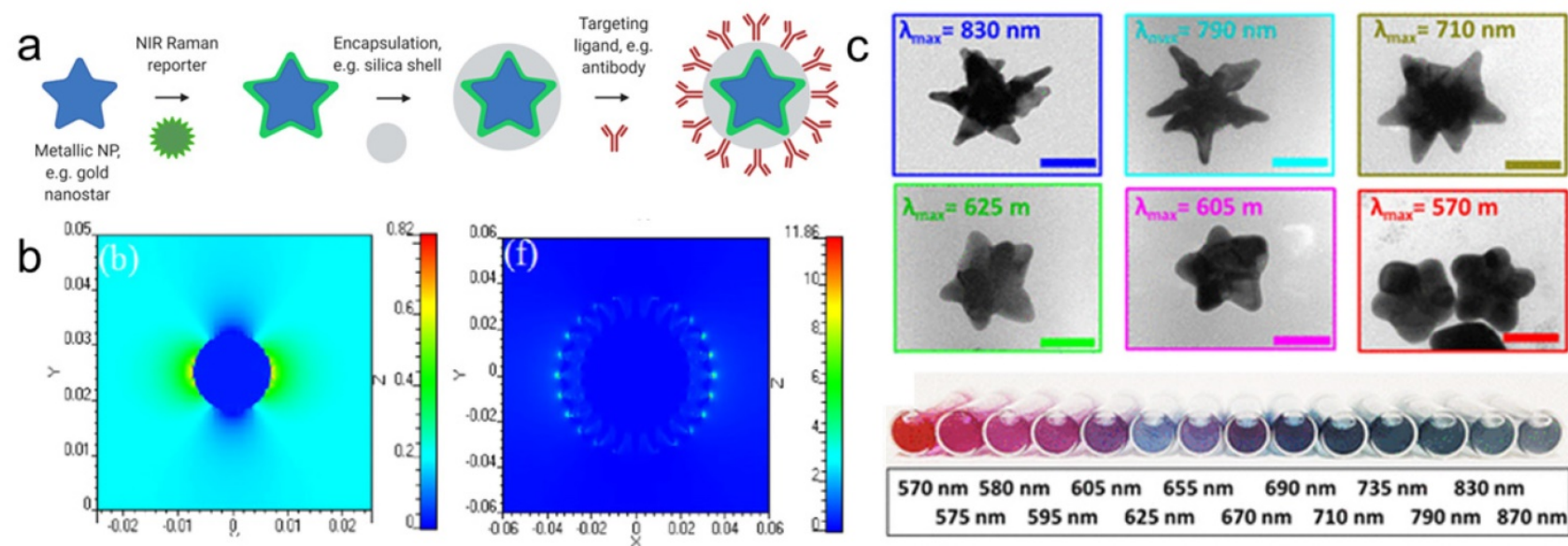

$570 \mathrm{~nm} 580 \mathrm{~nm} 605 \mathrm{~nm} 655 \mathrm{~nm} 690 \mathrm{~nm} 735 \mathrm{~nm} 830 \mathrm{~nm}$ $575 \mathrm{~nm} \quad 595 \mathrm{~nm} \quad 625 \mathrm{~nm} \quad 670 \mathrm{~nm} \quad 710 \mathrm{~nm} \quad 790 \mathrm{~nm} \quad 870 \mathrm{~nm}$

Figure 1. Schematic representation of SERRS nanotag structure for in vivo imaging applications. (a) Metallic substrates, e.g. gold nanostars, are functionalized with a NIR Raman reporter which are then encapsulated in a biocompatible coating such as silica. Active targeting can be achieved through conjugation of specific targeting moieties, e.g. antibodies to the nanoparticle surface. (b) 3D finite-difference time-domain (FDTD) simulated electromagnetic field distributions of a gold nanosphere (left) and a gold nanostar (right) excited with a $785 \mathrm{~nm}$ laser. Adapted with permission from ref. [16]. (c) TEM images (top) and color in solution (bottom) of gold nanostars with different LSPR maxima. Adapted with permission from ref. [17]. 
Table 1. Selected examples of gold nanostars synthesis methods.

\begin{tabular}{|c|c|c|c|c|}
\hline $\begin{array}{l}\text { Reducing } \\
\text { agent }\end{array}$ & $\begin{array}{l}\text { Auxiliary } \\
\text { shape-directing or } \\
\text { capping agent }\end{array}$ & $\begin{array}{l}\text { Seeds } \\
\text { presence }\end{array}$ & Comments & Reference \\
\hline Ascorbic acid & $\mathrm{CTAB}, \mathrm{Ag}^{+}$ & Seed-mediated & First example of nanostars synthesis, requires toxic CTAB. & {$[30]$} \\
\hline Ascorbic acid & $\mathrm{Ag}^{+}$ & Seed-mediated & Surfactantless nanostar synthesis allowing to obtain higher SERS signal. & [37] \\
\hline Ascorbic acid & - & Seedless & $\begin{array}{l}\text { Surfactantless, silver-free and seedless nanostar synthesis allowing to obtain highest SERS } \\
\text { signal. }\end{array}$ & {$[13,26]$} \\
\hline Ascorbic acid & - & Seed-mediated & $\begin{array}{l}\text { Surfactantless, silver-free and seed-mediated method of larger scale nanostar synthesis, allowing } \\
\text { to obtain highest SERS signal. }\end{array}$ & [42] \\
\hline $\mathrm{H}_{2} \mathrm{O}_{2}$ & - & Seed-mediated & $\begin{array}{l}\text { Surfactantless, silver-free and seed-mediated method that enabled shape-direction by tuning of } \\
\text { the reaction kinetics using } \mathrm{pH} \text { and } \mathrm{H}_{2} \mathrm{O}_{2}\end{array}$ & {$[72]$} \\
\hline Good's buffers & - & Seedless & High tip/core ratio, high polydispersity of obtained nanostars. & [56] \\
\hline Good's buffers & - & Seed-mediated & $\begin{array}{l}\text { Tip/core ratio and nanostars sizes can be controlled independently, high size and shape } \\
\text { monodispersity of the obtained nanostars. }\end{array}$ & [57] \\
\hline PVP & - & Seed-mediated & High monodispersity and shape/size control, however method requires toxic DMF as a solvent. & {$[64]$} \\
\hline PVP & - & Seed-mediated & $\begin{array}{l}\text { Highest known monodispersity and shape control of obtained nanostars grown from } \\
\text { monodisperse symmetrical seeds. Provide significantly higher SERS signal than asymmetric } \\
\text { nanostars. }\end{array}$ & [66] \\
\hline
\end{tabular}

Many different protocols of nanostars synthesis have been reported [26-28]. The outcomes and reproducibility of these synthesis protocols are affected by many factors, including, but not limited to the reducing, capping, and shape-directing agents used (with one reagent sometimes fulfilling multiple roles); the relative and absolute concentration as well as the oxidation state and ligands of the gold complexes used; the presence or absence of preformed seeds; reaction parameters like temperature, solvent and $\mathrm{pH}$ of the solution, scale of synthesis and mixing speed, and even the degree of glassware purity. Although it is highly desirable to obtain monodisperse nanoparticles during synthesis, it is possible to separate different shapes and sizes of nanoparticles by density gradient centrifugation [29] or field-flow fractionation. Below we will address the established gold nanostars synthesis methods and discuss their advantages and disadvantages with regards to their use in SERS-based bioimaging applications.

\section{Ascorbate-based synthesis}

Ascorbic acid is one of the most commonly used reducing agents in nanostars synthesis and was first used to produce nanostars in 2004 [30]. This initial protocol required cetyltrimethylammonium bromide (CTAB), a cationic, shape-directing surfactant that is highly cytotoxic [31]. Variations to this synthesis protocol have been reported where CTAB was replaced with sodium dodecyl sulfate (SDS) [32], albumin [33], benzylhexadecyldimethylammonium chloride (BDAC) and dioctyl sodium sulfosuccinate (AOT) [34], poly(diallyldimethylammonium chloride) (PDDA) [35], and nonionic surfactant Triton X-100 [36].

However, ascorbic acid's most important feature as a reducing agent is its ability to produce nanostars without any auxiliary surfactants. This is of great importance as stabilization of nanostars by auxiliary surfactants is achieved through adsorption to the NPs surface, which decreases the available surface area for Raman reporter adsorption and thus leads to diminished Raman signal intensity. In 2012, Vo-Dinh and co-workers described a surfactant-free gold nanostar synthesis protocol involving only gold seeds, gold ions, ascorbic acid, and small amounts of silver ions [37]. Silver ions functioned as a shape-directing agent and varying quantities resulted in the preparation of stars of different shapes with red-shifted LSPRs (Figure 2a). This protocol has since been modified in numerous other reports (Figure $2 b$ ) [38-40].

More recently, Kircher and co-workers presented a seedless and silver-free protocol, which produced gold nanostars through rapid addition of gold ions to a solution of ascorbic acid at reduced temperatures (Figure 2c) [13, 26, 41]. Since this method required rapid addition of gold ions to an ascorbic acid solution, it was difficult to scale-up; in larger volumes rapid homogeneous distribution of the gold ions across the entire volume was more difficult to achieve, leading to polydisperse nanostar preparations. This limitation was mitigated by using a seed-mediated version of ascorbate-based synthesis of gold nanostars (Figure 2d) [42].

\section{Silver as a shape-directing agent}

Silver ions $\left(\mathrm{Ag}^{+}\right)$are a commonly used ingredient and act as a shape-directing agent in the synthesis of branched plasmonic nanomaterials, including gold nanostars. As in the case of gold nanorod crystal growth, it is likely that $\mathrm{Ag}^{+}$ions can direct the anisotropic growth of gold nanoparticle seeds to form gold nanostars by preferential adsorption of silver halides onto the crystal facets of seeds, which restrict the growth of these passivated facets [43, 44]. Yuan et al. demonstrated that the growth kinetics of seed nanoparticles can be tailored during seed-mediated growth approaches by 
incrementally varying the $\mathrm{Ag}^{+}$concentration resulting in the formation of thorny three-dimensional (3D) nanoparticles [45]. In this case, $\mathrm{AgCl}$ formed in situ during the synthesis adsorbed on the seed crystal facets and facilitated the 3D anisotropic growth and formation of thorny gold nanostructures. The involvement of $\mathrm{Ag}^{+}$ions in anisotropic growth was further verified by the X-ray photoelectron spectroscopy (XPS) analysis [45]. It is also possible that poorly passivated defect sites and multiple twinning (crystal intergrowth or crystals sharing a facet) on the seed surface can play an important role in the anisotropic nanoparticle growth [46-48]. Atta et al. showed that $\mathrm{Ag}^{+}$ions play an important role in defining the crystallinity of the final nanostars and controls the number and length of thorns by directing the growth of the seed depending on seed twinning [49]. This facilitated a highly reproducible production of nanostars with high monodispersity and plasmon tunability [49]. While $\mathrm{Ag}^{+}$ions direct the anisotropic growth by passivating the seed twinning, a recent study showed that thermal treatment can increase the crystal twinning in the seed nanoparticles, which resulted in high yield production of nanoparticles with preselected morphologies [50]. This also provided an insight into the involvement of $\mathrm{Ag}^{+}$ions in passivating the twinned facets in seed nanoparticles.

Silver is also known for its role as an underpotential deposition (UPD) agent, being able to direct the anisotropic growth of gold nanoparticles via surface passivation [51-54]. In this mechanism, near-monolayers of $\mathrm{Ag}^{0}$ onto the growing nanoparticle surface can selectively adsorb on certain crystallographic facets, which restricts the growth of that crystal facet $[40,53]$. Even though various mechanisms explaining the role of silver in anisotropic crystal growth in plasmonic nanomaterials have been suggested, its precise role in the mechanism of the anisotropic crystal growth is still under debate.

\section{Good's buffers-based nanostar synthesis}

Good's buffers (e.g. HEPES, EPPS, MOPS, etc.) are generally zwitterionic water-soluble compounds with high biocompatibility and are widely used in biological research. The first report on the use of these buffers for nanostars synthesis dates back to 2005 [55]. It was demonstrated that nanostars could be produced with various shapes in these buffers by changing the $\mathrm{pH}$ and concentration of the Good's buffers. Xie et al. showed that the purity and homogeneity of the nanostars could be improved by controlling the temperature of the reaction [27]. Interestingly, while the authors concluded that MOPS buffer did not promote the formation of nanostars, Odom et al. later reported that MOPS can be used for nanostars growth in both seedless and seed-mediated synthesis conditions [56, 57]. The latter method is particularly interesting since it was demonstrated that by changing the concentration (Figure 3a) and size (Figure $3 b$ ) of the seeds, one can independently control the NPs core diameter and tip length. Furthermore, by varying $\mathrm{pH}$, the sharpness of nanostar spikes can also be controlled (Figure 3c). An additional dimension of shape tuning was added by Pompa and coworkers, who utilized hydroxylamine as a reducing agent, while HEPES was mainly used for shape direction [58].

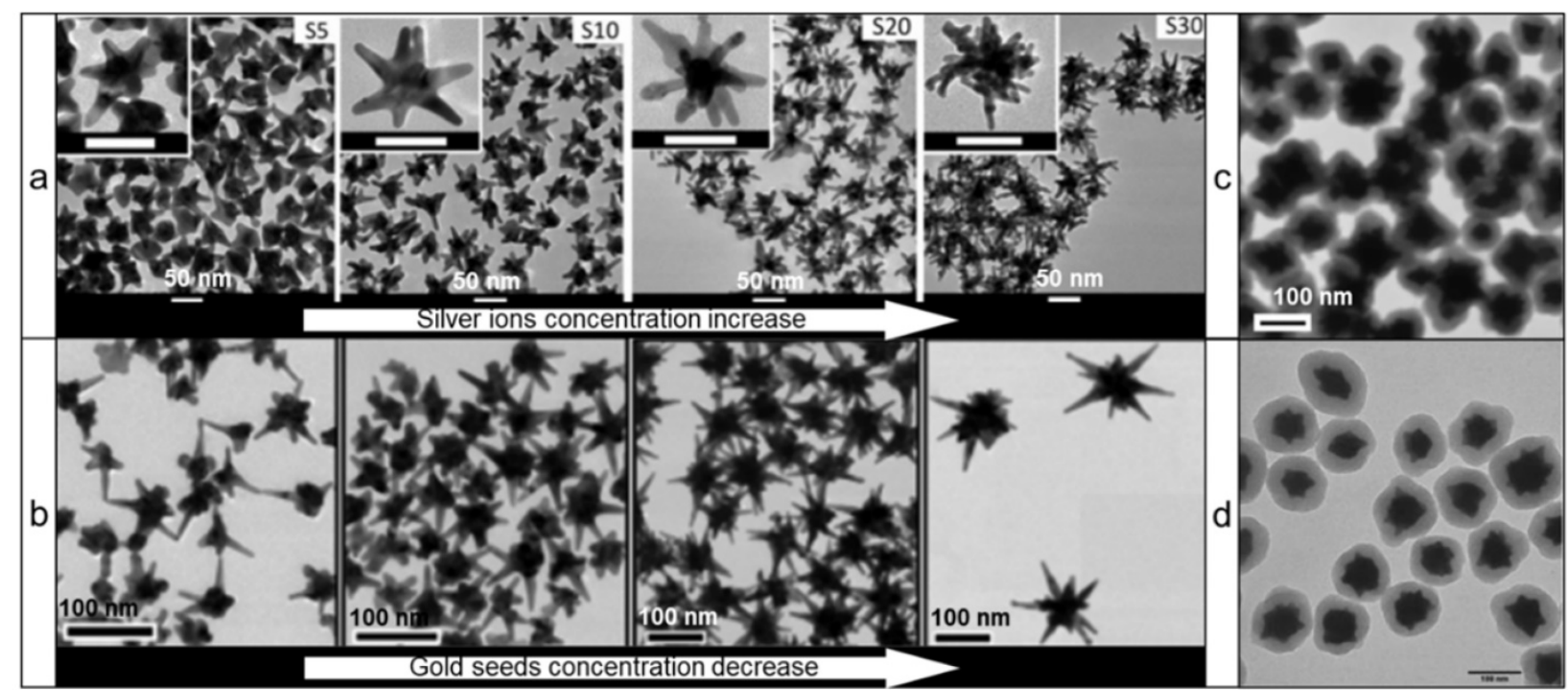

Figure 2. Transmission electron microscopy (TEM) images of various gold nanostars synthesized via surfactant-free protocols using ascorbic acid as a reducing agent. (a) Effect of silver concentration on the nanostar shape; adapted with permission from ref.[37] (b) Effect of gold seeds concentration on the nanostar shape; adapted with permission from ref.[40] Nanostars synthesized via (c) silver-free seedless and (d) seed-mediated protocols; adapted with permission from refs.[13, 42] 


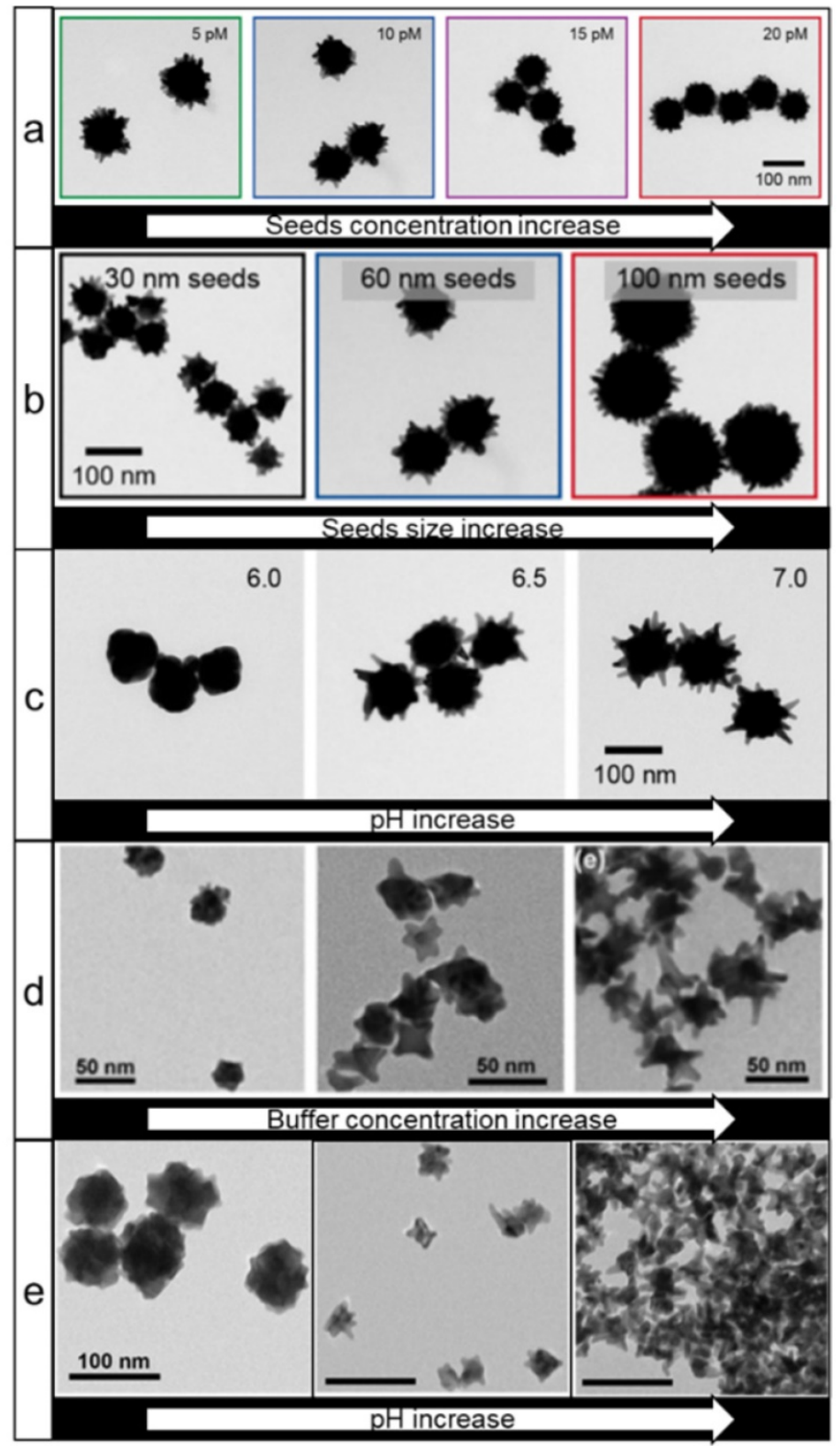

Figure 3. TEM images of gold nanostars prepared using Good's buffers. Effects of seeds concentration (a), size (b), and (c) pH increase on nanostars shape, adapted with permission from ref. [57], Copyright 2019 American Chemical Society. (d, e) Effects of buffer concentration and pH, respectively, on the nanostars size and shape during seedless synthesis, adapted with permission from ref. [59], Copyright 2014 American Chemical Society.

In contrast to seed-mediated methods, the seedless methods lack the ability to independently control sizes of core and tips without the use of auxiliary agents (Figure 3d, e) [56, 59]. However, by adding some silver ions during a seedless synthesis, nanostar shape control can be achieved [60]. A detailed investigation of the reaction mechanism showed that HEPES plays a dual role by binding to and stabilizing the surface of gold seeds via a complex, pH-dependent interplay between the nitrogen atoms and sulfonate groups, while reduction of gold ions onto the seeds occurs via oxidation of the nitrogen atoms to a cation radical as evidenced by electron paramagnetic resonance [61, 62].

\section{PVP-based nanostar synthesis}

Another well-established method used for the synthesis of nanostars relies on the use of polyvinylpyrrolidone (PVP) as both a reducing and stabilizing agent. This seed-mediated method produces nanostars in high yields and with high monodispersity [63]. Others demonstrated that the size, shape and thus LSPR of nanostars could be controlled by tuning the ratios of gold ions to gold seeds or by changing reaction temperature in the PVP/dimethylformamide (DMF) system (Figure 4a-c) $[64,65]$. An unprecedented degree of nanostar shape symmetry was achieved by the group of $\mathrm{Lu}$ who synthesized icosahedral gold seeds in diethylene- 
glycol (DEG)/PVP system and then grew nanostars from those seeds in a DMF/PVP system with the addition of various amines (Figure 4d) [66]. Interestingly, this symmetry granted a noticeable increase of SERS enhancement in symmetric nanostars relative to their more asymmetric counterparts.

Although these protocols are CTAB-free and are capable of producing high yields of monodisperse nanostars, their reliance on DMF (a highly toxic organic solvent) for synthesis is an undesirable feature for biomedical imaging applications. Moreover, they demonstrate high adsorption of PVP to the gold surface, which in turn decreases the overall Raman signal intensity since the PVP will compete with the Raman reporters for the nanostar surface [67].

\section{Surfactant-free methods}

In addition to the aforementioned synthesis procedures that either use toxic shape-directing surfactants or solvents (or combinations thereof), many efforts have been focused on designing syntheses that produce monodisperse nanostars in high yields under environmentally friendly conditions (i.e. aqueous conditions). Seed-mediated protocols using hydroquinone and citrate were reported in 2011, showing that $\mathrm{pH}$ and reagent concentrations determine the shapes of nanostars (Figure 5a) [68, 69]. A seedless synthesis of nanostars that employed glucosamine or glucamine without any additional surfactants or shape-directing agents was reported by Moukarzel et al., (Figure 5b) [70]. Similarly, homogeneous nanostars were produced by using gallic acid (Figure 5c) [71]. Kircher et al. developed a seed-mediated method to produce various shapes of nanoparticles using only alkaline hydrogen peroxide and gold chloride, demonstrating the capability to controllably generate various shapes and sizes of nanostars by closely tuning the reaction kinetics (Figure 5d) [72]. In another report, $\sim 400 \mathrm{~nm}$ star-like structures were obtained through the use of only dopamine as both the reducing and shape-directing agent [73] (Figure 5e). However, it was shown that by using a similar molecule, L-DOPA, one can also obtain nanostars of less than $100 \mathrm{~nm}$ size (Figure 5f) [74]. Using an adduct of tryptophan and glutaraldehyde, Ma and colleagues have synthesized nanostars of several different shapes and relatively large sizes (>200 nm; Figure 5g) [75]. Finally, star-like structures could also be obtained from solely star fruit juice and gold salt (Figure 5h) [76].

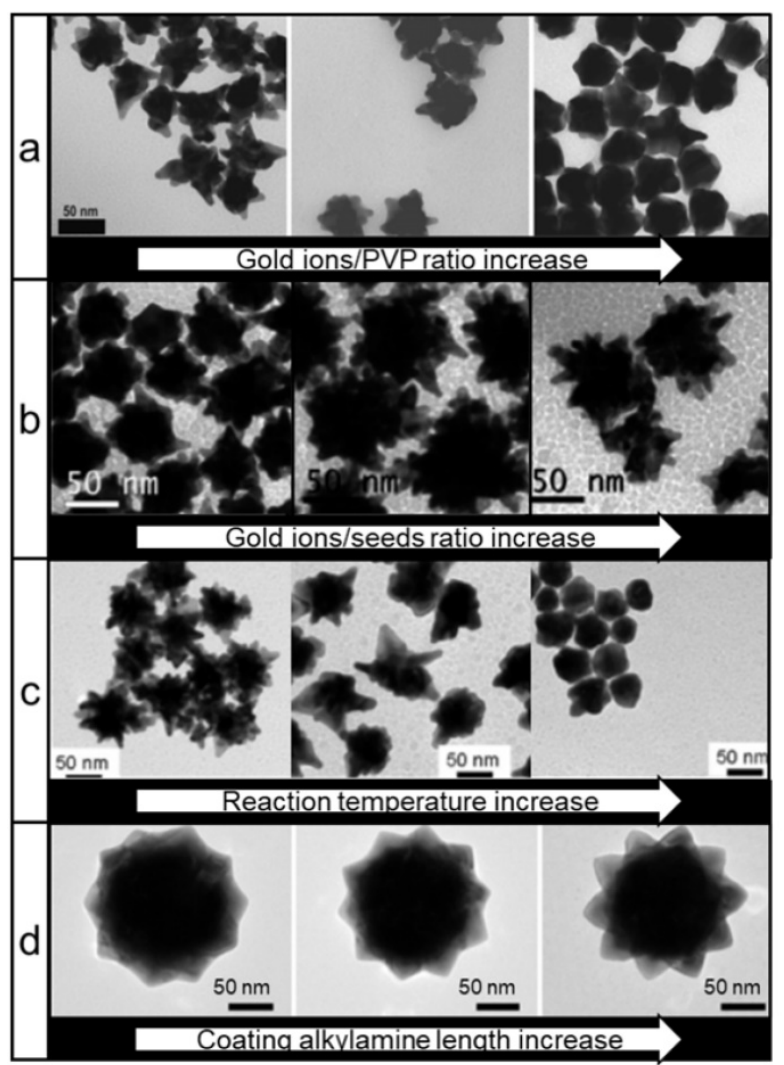

Figure 4. TEM images of different types of gold nanostars synthesized via PVP-based route. Nanostars shape control by changing: (a) gold ions/PVP ratio (adapted with permission from ref.[63]), (b) gold ions/gold seeds ratio (adapted with permission from ref.[64], Copyright 2010 American Chemical Society, (c) reaction temperature during synthesis (adapted with permission from ref.[64], Copyright 2010 American Chemical Society, (d) the alkyl chain length of an amine additive (adapted with permission from ref.[66], Copyright 2015 American Chemical Society. 


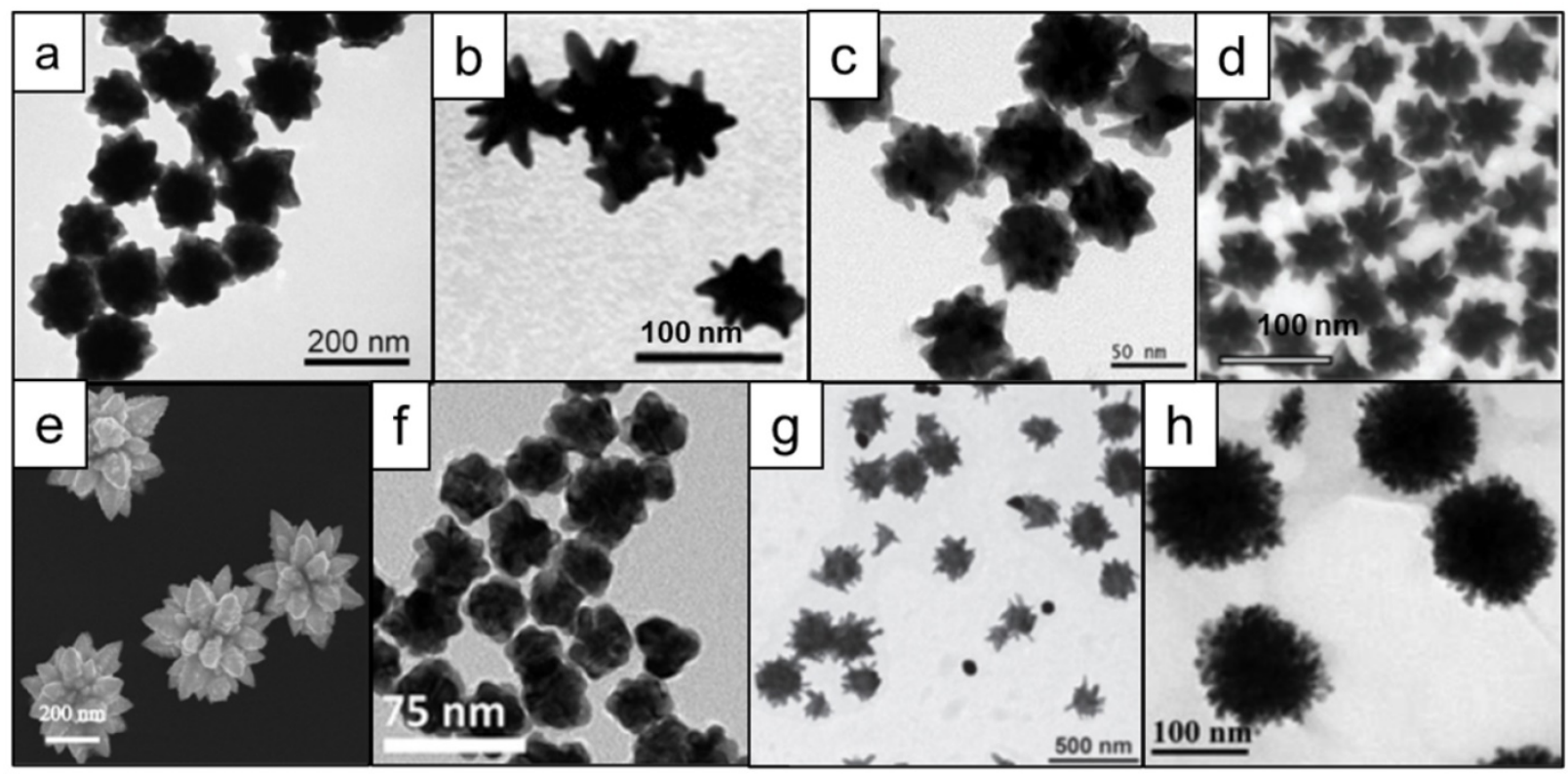

Figure 5. Microscopy images of different shapes of nanostars that can be obtained in surfactant-free conditions using various reducing agents. (a) hydroquinone, adapted with permission from ref. [68], Copyright 2011 American Chemical Society; (b) glucosamine, adapted from ref. [70] with permission from The Royal Society of Chemistry; (c) gallic acid [71], (d) hydrogen peroxide, adapted with permission from ref. [72], Copyright 2017 WILEY-VCH; (e) dopamine, adapted from ref. [73] under a Creative Commons Attribution (CC BY) license; (f) L-DOPA, adapted from ref. [74] with permission from The Royal Society of Chemistry; (g) tryptophan [75]; (h) star fruit juice, adapted from ref. [76] with permission from The Royal Society of Chemistry.

\section{Synthesis in microfluidic devices}

To improve the reproducibility of nanoparticles synthesis in terms of size, dispersity and morphology, in recent years more research has been focused on the use of microfluidic technology for nanoparticle preparation instead of bulk syntheses [77-80]. Microfluidic systems offer accurate control of various experimental parameters, such as sample volume, flow rate, mixing velocity, reaction time and temperature, providing a significantly more precise way to optimize the physical properties and hence imaging performance of SERS nanoprobes [78]. As such, microfluidic systems with different flow profiles, specifically continuous and segmented (or droplet) flows, have been used to produce a range of plasmonic nanostructures including gold nanostars which have been used as plasmonic cores of SERS nanoprobes [80-85].

Continuous flow microfluidic systems have enabled the seedless synthesis and functionalization of gold nanostars with an average size of about 50-70 $\mathrm{nm}$ and a high aspect ratio [84]. In a separate study, highly monodisperse gold nanostars averaging 60-80 $\mathrm{nm}$ in size were formulated using droplet microfluidics, either in the presence or absence of surfactants [85]. In contrast to the continuous flow microfluidic system, the droplet microfluidic platform offers improved mixing due to chaotic advection, narrower sample distribution, and reduced cross-contamination. As a result, higher quality gold nanostars with more controlled properties can be produced using droplet microfluidics.

Altogether, further advances in microfluidic technologies are anticipated to enable the fabrication of more complex (e.g, coated instead of just the metallic core) SERS nanoprobes on a single microfluidic platform (instead of using multiple microfluidic devices), as well as synthesizing increasingly complex SERS nanostructures with precisely regulated physicochemical properties.

\section{Choice of Raman reporter}

The choice of Raman reporter for a specific nanotag is as important as the choice of plasmonic core and depends on the surface properties of the metal nanoparticle core in terms of atomic composition ( $\mathrm{Au}, \mathrm{Ag}, \mathrm{Pt}$, etc.), zeta potential (positive or negative, absolute value), and the density of stabilizing agent. Independent of the plasmonic core, factors that are desirable for any Raman reporter include high Raman scattering cross-section (typically found in conjugated electron-rich polyenes or aromatic molecules like dyes); high photo- and chemical stability, molecular symmetry (to reduce the number of peaks in a Raman spectrum while increasing their relative intensity); absorption maximum in resonance with the excitation laser wavelength, and having anchoring groups with high affinity to the nanoparticle surface (e.g. thiols, disulfides, thiophenes, isothiocyanates, etc.) [86-89]. Below we will discuss design considerations of Raman reporters to optimize Raman signal intensity of SE(R)RS nanotags. 


\section{Selected aromatic thiols used as Raman reporters}

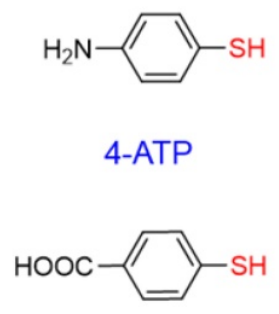

4-MBA

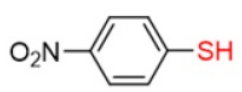

4-NTP

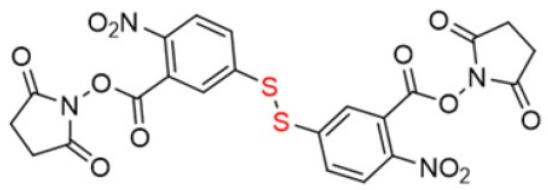

DSNB
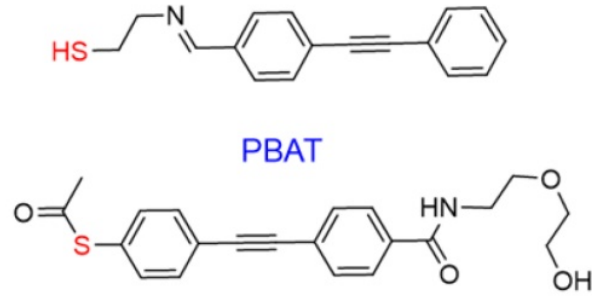

SEMA4

Figure 6. Chemical structures of aromatic thiols used as Raman reporters in SERS nanotags. Commonly used commercial reporters 4-NTP, 4-MBA, and 4-ATP and selected designer reporters SEMA4 [96], PBAT[97], and DSNB[98]. "Anchoring" sulfur atoms are shown in red.

\section{Aromatic thiols}

Interest in aromatic thiols as Raman reporters arises from their ability to form strong gold-sulfur bonds,[90] which leads to high gold surface coverage by such molecules [91]. Popular examples of such reporters include 4-nitrothiophenol (4-NTP) [92, 93], 4-aminothiophenol (4-ATP)[93, 94], and 4-mercaptobenzoic acid (4-MBA) [39, 95]; all of which are simple aromatic thiols with minimal structural complexity leading to clean Raman spectra and potential for further conjugation.

Even though the vast majority of these molecules are commercially available, many "designer" Raman reporters have been synthesized to include additional functionalities (Figure 6). For example, small anchoring molecules containing triple bonds like SEMA4 or PBAT produce intense Raman scattering signal in the silent Raman region - a region found between $1750-2750 \mathrm{~cm}^{-1}$ where background signal from biomolecules is reduced [96, 97]. DSNB was designed as a linker to attach antibodies to the gold surface, but also demonstrated favorable properties as a Raman reporter [98]. Despite dense surface coverage, these molecules have absorption maxima in the 200-300 $\mathrm{nm}$ range and therefore are not in resonance with NIR lasers leading to reduced Raman scattering cross-sections.

\section{Organic dyes}

Rhodamine 6G became very popular due to its large cross-section and has been widely used in SERS enhancement factor studies [6, 99, 100]. Triarylmethine dyes, such as malachite green or crystal violet are also often used in SERS studies due to a combination of their large cross-section, negligibly low fluorescence and low price. Malachite green isothiocyanate is the most popular of the family due to the presence of the isothiocyanate anchoring group that provides higher affinity to the gold nanoparticle surface relative to other dyes [101, 102].

The most attractive Raman reporters for in vivo studies are NIR-absorbing dyes due to their resonant Raman signal enhancement when irradiated with a NIR laser. The majority of these dyes are based on cyanine 7 or 7.5 backbone, with different substituents granting tunability in absorption maxima position and Raman spectral fingerprints. Common examples of these dyes are IR 792 [42, 103-105], IR 780p [106, 107], IR 780i [104, 108, 109], and DTTC [101, 103, 110, 111], which is often utilized as a benchmark dye for SERS intensity comparison studies [112].

Although multiple studies performed the comparison of SERS signal intensity of several commercial dyes, the Raman signal intensity is highly dependent on the type of plasmonic core and experimental conditions used, which complicates the interpretation of these results between different studies $[103,104,113,114]$. While some reports use the Raman signal intensity of a common chemical such as ethanol [103] as a benchmark (e.g. SERS nanotags made with dye A produce $X$ times higher Raman signal than ethanol, while the same nanotags made with dye $B$ produce $Y$ times higher signal), currently there is no universal standardized protocol for reporting SERS signal enhancements that enables the uniform comparison of signal intensities between different groups. 


\section{Commonly used commercial non-NIR Raman reporter dyes}<smiles>[R6]Oc1cccc(C(=O)OCC)c1-c1c2cc(C)c(=[NH+]CC)cc-2oc2cc(NCC)c(C)cc12</smiles>

Commonly used commercial NIR Raman reporter dyes

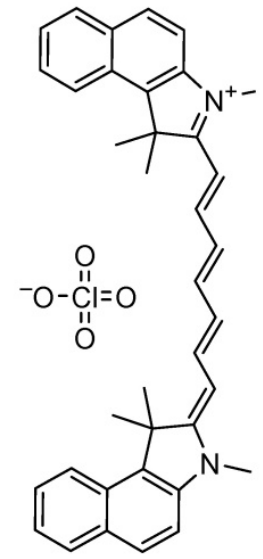

IR 780 perchlorate

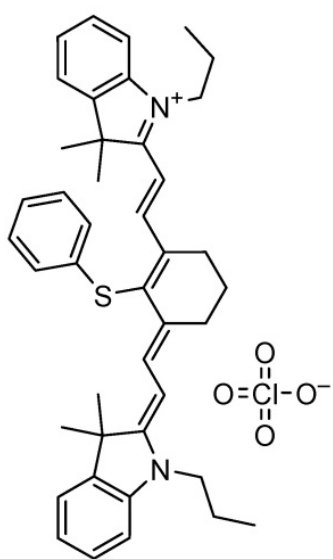

IR 792

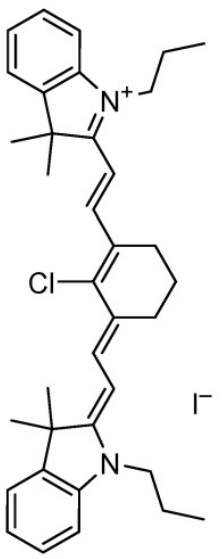

IR 780 iodide

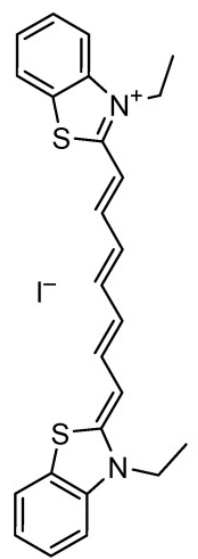

DTTC

Figure 7. Chemical structures of popular commercial organic dyes used as Raman reporters in SERS nanotags. "Anchoring" sulfur atoms are shown in red.

\section{Rational Raman Reporter Design}

Raman reporters often require specific design characteristics that are not found in commercially available dyes. As such, a lot of efforts have been focused on the design of Raman reporters that have a high affinity for the gold nanoparticle surface and are resonant with the excitation laser. For example, by adding a thiol group to $\beta$-carotene (Figure 8a), Kneipp et al. demonstrated a 9-fold increase in SERS signal in comparison to the parent molecule [115]. Similar polyene molecules were synthesized by Keller et al. (Figure 8b) [116]. Adding thiol groups to the xanthene ring of a rhodamine in an attempt to increase its anchoring abilities has been reported, but the designer dye CRh-SH (Figure 8c) failed to outperform commercial rhodamine 6G [117]. The group of Chang published a library of triphenylmethine dyes containing lipoic acid residue, with the brightest dye being B2LA (Figure 8d)[118]. However, since that family of dyes was not in resonance with biologically relevant $785 \mathrm{~nm}$ laser, they later developed 80 more dyes derivatized with lipoic acid [112]. In this instance the dye scaffold was based on the NIR dye cyanine 7 . Their dye CyNAMLA-381 (Figure 8e) produced a 12 times higher Raman intensity relative to the DTTC under similar experimental conditions. A cyanine 7-lipoic acid conjugate (Cy7-lip; Figure 8f) was shown to outperform conventional rhodamine $6 \mathrm{G}$ by more than 10 times [119]. A similar approach of adding a lipoic acid residue for anchoring the dye to the gold nanoparticle surface was demonstrated by the group of Maiti with a squaraine dye and a tetraphenyl ethylene (TPE) derivative (Figure 8g, h) [120, 121]. Another dye developed by the same group belongs to a NIR family of aza-BODIPY dyes (Figure 8i) and was reported to have significantly higher Raman intensity than crystal violet or rhodamine B [122]. The bidentate lipoic acid-conjugated squaraine dye M1 (Figure 8j), developed by the group of Liu, was shown to permit the selective growth of silver nanospheres on the tips of gold nanostars [123]. IR783B, reported by Yue et al., is a cyanine dye containing a thiol moiety for anchoring purposes and is resonant with $785 \mathrm{~nm}$ laser (Figure 8k) [124]. A more unconventional approach to a NIR Raman reporter design was chosen in a collaborative effort between the groups of Kircher and Detty, who, instead of adding a sulfur-containing linker to an existing dye, synthesized chalcogenopyrylium-based chromophores containing sulfur in the 
dye core (Figure 81). The corresponding SERRS nanotags demonstrated the highest Raman signal sensitivity to date, which was in the attomolar range [12]. Furthermore, this Raman reporter type can be further red-shifted by substituting the sulfur for selenium atoms and/or extending the conjugation chain to allow these dyes to be resonant with even longer wavelength excitation lasers such as 850 and even $1064 \mathrm{~nm}[125,126]$.

\section{Coating types}

To protect the metal-molecule system from environmental factors that could lead to decreased colloidal stability and/or desorption of Raman reporters from the metal core, it is embedded in an encapsulant layer. The most commonly used encapsulants include silica, synthetic polymers, and biomolecules such as proteins or lipids, and sometimes a composite shell can include a combination of these components.

\section{Designer Raman reporter dyes}

a<smiles>CC1=C(/C=C/C(C)=C/C=C/C(C)=C/C=C/C=C(C)/C=C/C=C/c2ccc(CS)cc2)C(C)(C)CCC1</smiles>

C<smiles>Nc1ccc2c(-c3ccc(C(=O)O)cc3C(=O)O)c3ccc(=[NH2+])c(S)c-3oc2c1S</smiles>

f<smiles>CC1(C)C(/C=C/C2=C(Cl)/C(=C/C=C3/N(CCO)c4ccccc4C3(C)C)CCC2)=[N+](CCOC(=O)CCCCC2CCSC2)c2ccccc21</smiles>

$\mathrm{h}$<smiles>CC1(C)C(/C=C/c2ccc(C(=C(c3ccccc3)c3ccccc3)c3ccccc3)cc2)=[N+](CCCNC(=O)CCCCC2CCSS2)c2ccc3ccccc3c21</smiles><smiles>CCOC(=O)/C=C/C=C/C=C/C=C/C=C/c1ccc(SC(C)(C)C)cc1</smiles><smiles>CN(C)c1ccc(C(=C2C=CC(=[N+](C)C)C=C2)c2ccc(NC(=O)CCCCC3CCSS3)cc2)cc1</smiles><smiles>CCCN1/C(=C/C=C2\CCCC(/C=C/C3=[N+](CCCNC(=O)CCCCC4CCSS4)c4ccccc4C3(C)C)=C2N(Cc2ccc(OC)cc2)C(C)=O)C(C)(C)c2ccccc21</smiles>

g<smiles>CC1(C)C(/C=C2\C(=O)C(/C=C3/N(CCCNC(=O)CCCCC4CCSS4)c4c(ccc5ccccc45)C3(C)C)=C2O)=[N+](CCCNC(=O)CCCCC2CCSS2)c2ccc3ccccc3c21</smiles>

i<smiles>COc1cc(OC)cc(C2=CC(c3ccc(N)cc3)=NC2=Nc2c(-c3cc(OC)cc(OC)c3)cc(-c3ccc(N)cc3)n2PF)c1</smiles>

j

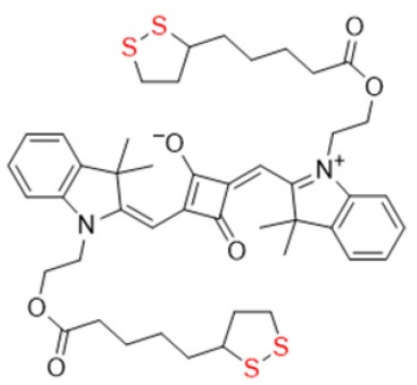

k

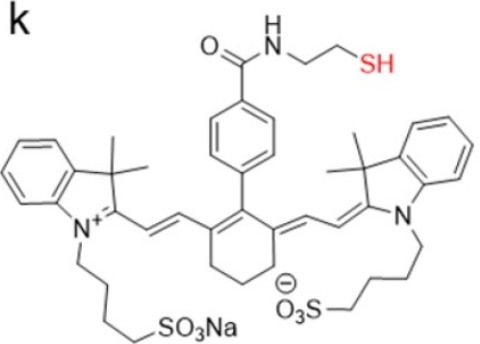

I

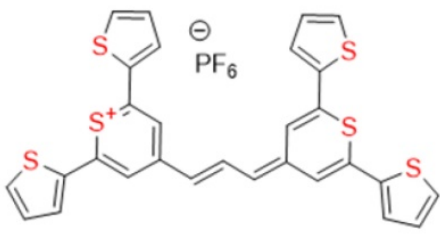

Figure 8. Chemical structures of organic dyes designed to be reporters for SERS nanotags. (a) Carotenethiol[115], (b) polyenethiol[116], (c) thiolated rhodamine[117], (d) B2LA[118], (e) CyNAMLA-381 [112], (f) Cy7-lip[119], (g) SQ3[120], (h) TPE-In-L[121], (i) aza-BODIPY[122], (j) MI [123], (k) IR783B[124], (l) chalcogenopyrylium dye[127]. "Anchoring" sulfur atoms are shown in red. 
Table 2. Selected examples of SERS nanotags coatings.

\begin{tabular}{|c|c|c|}
\hline Coating type & Comments & Reference \\
\hline PEG & First example of successful in vivo tumor imaging using SERS nanotags covered by PEG layer attached directly to gold surface. & [101] \\
\hline PEG, silica & $\begin{array}{l}\text { While silica coating prevents Raman reporter from detaching from gold surface, PEG layer increases nanoparticles blood } \\
\text { circulation time and decreases liver and spleen uptake. }\end{array}$ & {$[12,13,26,132$} \\
\hline PDA & $\begin{array}{l}\text { PDA coating not only granted SERS nanotags biocompatibility, but also allowed bone microfractures imaging due to high } \\
\text { affinity of PDA towards calcium ions. }\end{array}$ & [136] \\
\hline $\begin{array}{l}\text { PMA-dodecylamine } \\
\text { amide }\end{array}$ & $\begin{array}{l}\text { Amphiphilic nature of the coating polymer allowed nanotags with high surface coverage by hydrophobic Raman reporters } \\
\text { become hydrophilic and colloidally stable in water. }\end{array}$ & [138] \\
\hline $\begin{array}{l}\text { PEG, } \\
\text { PMMA-co-4VP }\end{array}$ & $\begin{array}{l}\text { Due to } \mathrm{pH} \text {-sensitive nature of PMMA-co-4VP coating polymer, the multinanoparticle liposomal SERS construct could be } \\
\text { disassembled in lysosomes following cellular uptake, allowing effective monitoring and controlled release of cargo. }\end{array}$ & [140] \\
\hline $\begin{array}{l}\text { Polyaniline } \\
\text { Polypyrrole / PVP }\end{array}$ & $\begin{array}{l}\text { Conjugated polymers for nanoparticles coating used in the study provided a viable alternative to organic dyes as SERS } \\
\text { reporters, however they required additional coating with PVP to increase colloidal stability. }\end{array}$ & [142] \\
\hline $\begin{array}{l}\text { PNIPAm-co-AAm } \\
\text { PEG }\end{array}$ & $\begin{array}{l}\text { By coating drug-loaded branched gold nanoshells with thermoresponsive PNIPAm-co-AAm with a Raman reporter at a } \\
\text { terminal end, it was possible to monitor photothermal heating and drug release from the nanoparticles by increase in SERS } \\
\text { signal due to polymer collapse. }\end{array}$ & [146] \\
\hline Polystyrene & $\begin{array}{l}\text { Remarkably stable polystyrene coating for SERS nanotags allowed their storage for } 6 \text { months without significant loss of SERS } \\
\text { intensity. }\end{array}$ & [147] \\
\hline Silica & $\begin{array}{l}\text { First primer-free silica coating protocol that allowed to achieve unprecedentedly high SERS intensities due to absence of } \\
\text { competition for surface for the Raman reporter. }\end{array}$ & [26] \\
\hline Silica & Ultrasound-assisted silica coating of nanoparticles can increase the shell stability towards hydrolysis. & [160] \\
\hline BSA & $\begin{array}{l}\text { BSA coating was shown to better protect gold nanostars from reshaping into spheres and aggregation than PEG coating after } \\
\text { storage for } 24 \text { hours at room temperature. }\end{array}$ & [104] \\
\hline DNA & $\begin{array}{l}\text { Coating SERS nanotags with oligonucleotides was demonstrated to not only increase the particles colloidal stability, but also } \\
\text { allowed simultaneous imaging and quantification of multiple miRNAs inside cells in vitro. }\end{array}$ & {$[170]$} \\
\hline Lipids & $\begin{array}{l}\text { Lipid membrane coating of gold nanoparticles allows encapsulation of multitude of different Raman reporters inside it, } \\
\text { removing the requirements for Raman reporters to have anchoring groups or charges. }\end{array}$ & [173] \\
\hline
\end{tabular}

\section{Polymer coating}

Polymers have been widely used as stabilizing agents. They allow for a high degree of flexibility, because their properties that include biodegradability rate, hydrophobicity, or polymer chain packing, can be finely tuned by altering their chemical structure and ultimately dictate physicochemical properties of the SERS nanotags [128].

The most widely used polymer in the fabrication of nanoparticles for biomedical applications is polyethylene glycol (PEG) - it is highly hydrophilic, chemically inert, and has antifouling properties to prevent opsonization of biomolecules on the nanoparticle surface, providing "stealth-like" properties leading to increased circulation times [129, 130]. Nie et al. were the first to produce injectable SERS nanotags that constituted spherical gold nanoparticles coated with Raman reporters that were stabilized by thiolated-PEGs [101]. This PEG layer can be attached both to the gold surface directly [95, 101, $131]$ and to another coating layer [132, 133].

Polydopamine (PDA) is another biocompatible polymer used to stabilize gold nanoparticles [134, 135]. It is formed in situ during nanoparticle surface coating with molecular dopamine. Due to its high affinity towards calcium ions, PDA was used to create SERS nanotags which specifically targeted bone microfractures after either intravenous or intramuscular injection [136].

Amphiphilic polymers have been utilized extensively in NPs coating strategies due to their ability to adsorb onto hydrophobic surfaces and render them hydrophilic [137]. Such properties of the amide conjugate of polyisobutylene-alt-maleic anhydride (PMA) and dodecylamine were highlighted by Liz-Marzán, where the polymer was used for the coating of SERS nanotags with a high surface coverage of hydrophobic Raman reporters [138]. As was demonstrated by Chen et al., amphiphilic copolymers (such as polystyrene-block-poly(acrylic acid) in this instance) are compatible not only with various Raman reporters but also with nanoparticles of different shapes and sizes [139].

Combining amphiphilicity with $\mathrm{pH}$-sensitivity of coating polymers allowed Song et al. to create plasmonic vesicles made entirely of self-assembled gold nanoparticles that incorporated a high number of hotspots [140]. First, $14 \mathrm{~nm}$ gold NPs were independently coated with both hydrophilic PEG chains and a hydrophobic copolymer of 4-vinylpyridine (4VP) and methyl methacrylate (MMA). Then these small nanoparticles were self-assembled into $100-200 \mathrm{~nm}$ vesicles which were internalized by cells and disassembled in lysosomes specifically due to the $\mathrm{pH}$-sensitive nature of their hydrophobic copolymer coating component (Figure 9a). Moreover, attaching PEG to the gold surface via the acid-responsive hydrazone linker allowed the group of Li to develop SERRS nanotags that could cross the blood-brain barrier and then self-assemble inside tumors to create hotspots and dramatically enhance their Raman signal (Figure 9b) [141]. 

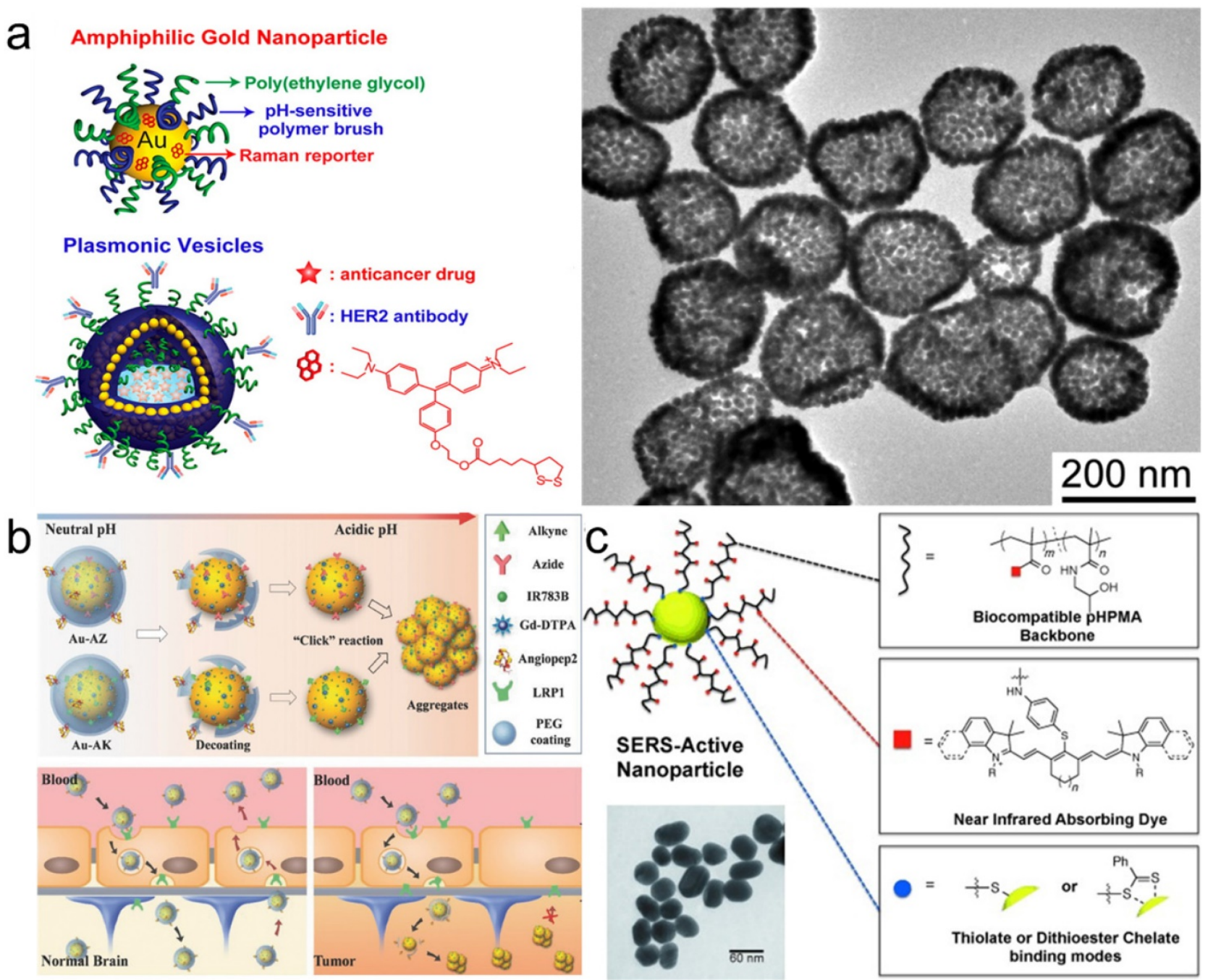

Near Infrared Absorbing Dye

Figure 9. Polymer-coated SERS nanotags. (a) Schematic illustration (left) and TEM image (right) of plasmonic vesicles self-assembled from dual polymer-coated SERS nanotags, adapted with permission from ref. [140], Copyright 2010 American Chemical Society; (b) PH-responsive PEG-coated nanotags which self-assemble inside brain tumors, adapted with permission from ref. [141], Copyright 2017 WILEY-VCH; (c) SERS nanotags with a dye-conjugated polymer coating for dual SERS-fluorescence imaging of lymph nodes, adapted with permission from ref. [143], Copyright 2014 WILEY-VCH.

Since polymers compete with the Raman reporter for the gold nanoparticle surface, this can lead to uneven or diminished loading of the Raman reporter, resulting in decreased Raman signal. To address this issue, NIR-absorbing polymers, such as conducting polymers polyaniline or polypyrrole, were used as Raman reporters. Liu et al. demonstrated that these polymers coated onto gold nanorods exhibit strong resonant Raman scattering enhancement when irradiated with biologically relevant $785 \mathrm{~nm}$ laser [142]. Another way to combine reporter and coating was demonstrated by Iacono et al., who synthesized a thiol-terminated poly(N-(2hydroxypropyl)methacrylamide and covalently conjugated NIR dyes throughout the polymer chain (Figure 9c)[143]. This NIR reporter-containing coating not only stabilized the NPs, but also allowed to perform both Raman and fluorescence imaging to detect lymph nodes.
Microgel-forming and thermoresponsive poly(N-isopropylacrylamide) (pNIPAM) was used as a coating, but in this instance Raman reporters were added after polymer attachment - due to its porous nature, reporters could diffuse inside the microgel and reach the gold surface [144]. Subsequently, the pores were "sealed" through a layer-by-layer assembly of poly(allylamine hydrochloride) and poly(acrylic acid). The thermo-responsive nature of pNIPAM was highlighted by Kearns et al. who created a hollow nanogold-based SERS nanotag coated with pNIPAM having a Raman reporter on the distal end of the polymer [145]. Such nanotags changed the SERS signal intensity upon laser irradiation due to photothermal heating, because pNIPAM brought the reporter closer to the gold surface upon collapsing. A similar approach, but with a thermo-responsive copolymer of pNIPAM and acrylamide was utilized by Song et al. to coat branched gold nanoshells [146]. 
Upon heating to $40^{\circ} \mathrm{C}$ this polymer collapsed, which was shown by the increase in the rhodamine B Raman scattering that was attached to it. Such response to heating was used to load the nanoshells with drugs and subsequently release them upon laser irradiation.

Even though stability of nanotags during storage is a valid concern, using polystyrene for coating allowed $\mathrm{Yu}$ et al. to create extra stable nanotags with minimal SERS signal loss upon storage up to half a year in solution [147].

\section{Silica coating}

Primary reasons for the popularity of silica as an encapsulating layer include chemical inertness, mechanical rigidity, biodegradability, lack of contribution to the nanotag Raman spectrum and high degree of tunability [148, 149]. Shell thickness, porosity, chemical stability and biodegradability can be tuned depending on the desired application. However, not all silication protocols are suitable for yielding high intensity SERS nanotags, and below we will discuss the evolution of strategies used to develop protocols for the brightest nanotags.

The first report of silica coating of gold nanoparticles dates back to 1996 and describes a multi-step process, which includes: 1 ) gold nanoparticle surface priming with (3-aminopropyl)trimethoxysilane (APTMS) or (3-mercaptopropyl)trimethoxysilane (MPTMS), 2) hydrolyzing the APTMS/MPTMS layer in the presence of sodium silicate to create a thin $(2-4 \mathrm{~nm})$ silica shell over $24 \mathrm{~h}$ and finally 3$)$ growing thicker silica shell using classical Stöber method [150] - hydrolysis of tetraethyl orthosilicate (TEOS) by ammonia in aqueous ethanol (Figure 10a) [151, 152]. Later this protocol was adapted for synthesizing SERS nanotags by adding the dropwise addition of the
Raman reporter between steps 2 and 3 [153]. Considering that both the surface primer and the Raman reporter compete for the gold nanoparticle surface, it was important to find an optimal ratio that enabled the maximum amount of Raman reporter to adsorb to the gold nanoparticle surface whilst ensuing enough primer was present to render the surface vitreophilic. Even though earlier reports required the Raman reporter dye to have an anchoring group, such as isothiocyanate [152], this requirement was later rectified [154] (Figure 10b). Alternatively, surface priming is performed with PVP from which the silica shell is grown using a Stöber method [155]. To improve surface coverage of the Raman reporter, a consecutive coverage with a monolayer of the high affinity Raman reporter DTNB, polyallylamine and PVP was performed by Schlücker and co-workers before growing the silica shell [156]. The same group also demonstrated that a conjugate of APTMS and mercaptobenzoic acid acted both as a reporter and a primer for silica growth [157]. Typically, silication is performed in alcohols, which destabilizes the nanoparticles leading to aggregation. To increase nanoparticle stability against precipitation in ethanol during the silication process, Mir-Simon et al. utilized mercaptoundecanoic acid as a surface primer and demonstrated it was less detrimental to the Raman reporter signal than PVP [158]. Similarly, Fales et al. stabilized gold nanoparticles with thiol-PEG to prevent aggregation of the gold nanostars during subsequent silica coating in the presence of DTTC [159]. A drawback of using capping agents such as thiol-PEG is that these agents have a high affinity for the gold nanostar surface and outcompete low affinity Raman reporters such as DTTC.

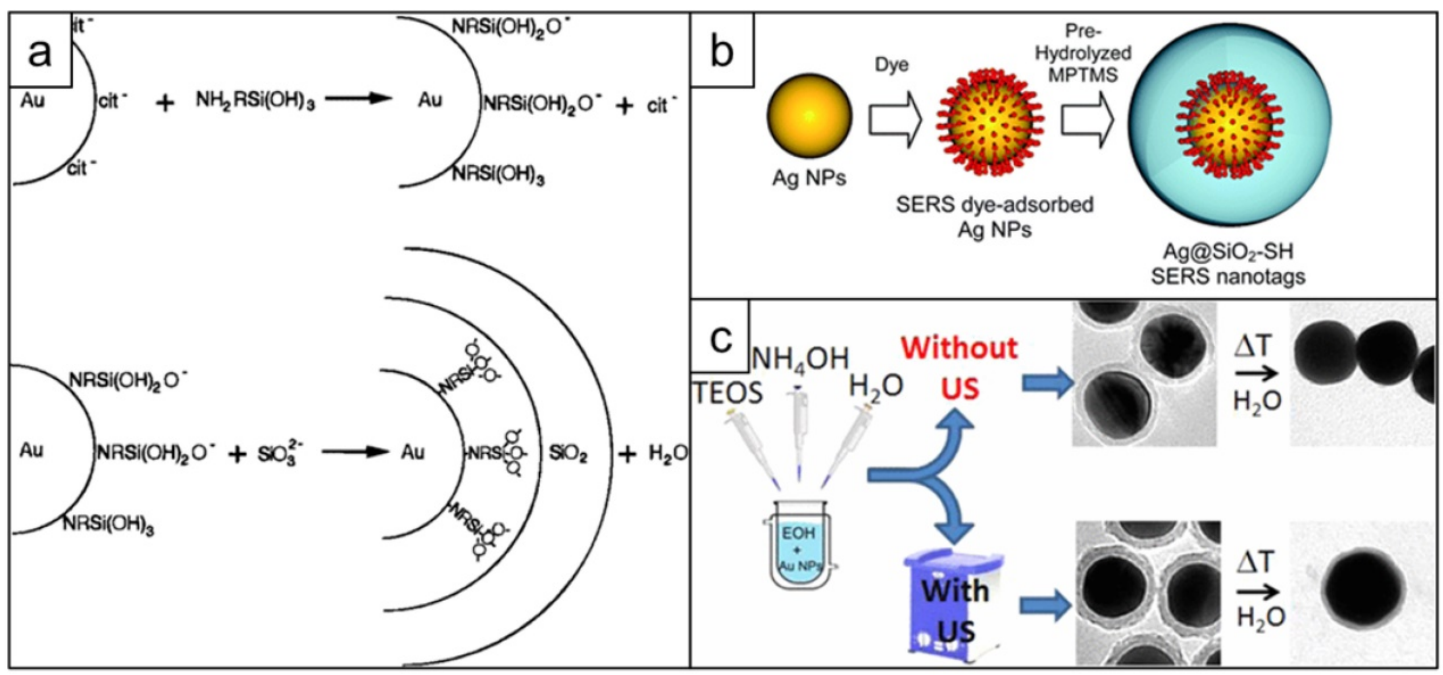

Figure 10. Development of silica coating for SERS nanotags. (a) Schematic representation of the surface reactions taking place during first successful two-step silica coating of gold nanoparticles, adapted with permission from ref. [151], Copyright 1996 American Chemical Society; (b) Scheme showing one step silication of a SERS nanotag, adapted from ref. [154] with permission from The Royal Society of Chemistry; (c) Stabilizing effect of applying sonication during nanotag silication, adapted with permission from ref. [160], Copyright 1996 American Chemical Society. 
Ideally, a primer-free silication method that is compatible with a wide variety of Raman reporters would be used. A protocol fulfilling this need was published by the Kircher group. A primer-free synthesis protocol of SERRS nanostars was developed, which, due to the lack of competition for the nanoparticle surface, produces unprecedented SERRS signals [13, 26, 127]. Others demonstrated that ultrasonication could be used during the synthesis of silica shells to significantly improve their stability towards hydrolysis (Figure 10c) [160].

\section{Biomolecular coating}

\section{Proteins}

Bare metallic nanoparticles coming into contact with the biological environment are known to adsorb surrounding proteins, forming what is called a "protein corona" [161, 162]. However, selective precoating of the NPs with proteins allows more control over the protein corona composition, and as a result, nanoparticle properties [163]. One of the most popular choices for this approach is bovine serum albumin (BSA). Coating of the nanotag surface with BSA is a rapid and straightforward approach to enhance the colloidal stability and biocompatibility of the nanotags. BSA was used, for example, in the preparation of Raman-based $\mathrm{pH}$ sensors [164] and dual SERS-upconversion fluorescence nanotags [165]. Glutaraldehyde-crosslinked BSA was utilized by Samanta et al. as a more stable version of BSA coating for their series of SERS nanotags [112]. Moreover, there are reports showing that BSA coating can prevent NP aggregation more effectively than PEG coating [104]. As shown by Blanco-Covián et al., antibodies can also be used as a stabilizing layer when they are attached directly to the gold surface [166].

\section{Nucleic acids}

Owing to their high hydrophilicity, DNA aptamers can be used not only for targeting but also as a stabilizing coating layer. For example, in a report by Wang et al. the authors directly attached thiol-terminated DNA aptamers to the surface of gold nanoparticles, which allowed them to develop an apta-immunoassay for cancer exosomes detection [167]. Another assay was developed for prostatespecific antigen detection by Jiang and coworkers based on DNA-covered SERS nanotags [168]. Using SERS nanotags with polycytosine $\mathrm{Li}$ et al. created a sensor which increases its Raman signal intensity upon $\mathrm{Hg}^{2+}$-induced nanotag aggregation [169]. Different thiol-terminated DNA sequences were also used by Tang and coworkers to coat SERS nanotags for the quantification of miRNA in living cells [170]. Another application of DNA-coated SERRS tags was demonstrated by Simoncelli et al. who developed optothermally tunable plasmonic nanoantennas capable of single-molecule detection [171].

\section{Lipids}

The main advantages of lipids as coatings include their inherent biocompatibility and highly predictable nature of self-assembly based on the composition of lipid mixture. The first lipid-encapsulated SERS nanotags were developed in 2010 by the Zheng group [172]. They showed that by using a mixture of single- and double-chain phospholipids one could encapsulate Raman reporter-coated gold nanoparticles inside lipid bilayer without the use of sulfur-containing surface primers and losing Raman signal intensity. Later on, Walker and colleagues published their protocol for the synthesis of SERS nanotags with a lipid coating, which utilized a different lipid bilayer composition and was compatible with three different types of Raman reporters [173]. The same group used the developed phospholipid coating to demonstrate controlled tip-to-tip aggregation of gold nanorods, creating strong hotspot SERS enhancement in the junction [174]. Su et al. [175] exploited the dynamic nature of lipid bilayers to demonstrate the environmental sensitivity of their SERS nanotags. In this instance, depending on the Raman reporter used, the Raman signal changed in response to heating, presence of other lipid bilayers or the presence of surfactants. A lipid-porphyrin conjugate coating was developed by the group of Zheng,[176] which combined extra high loading of the Raman reporter inside lipid bilayer with stabilization it provides. A relatively unconventional synthetic approach was chosen by the group of Kang,[177] who instead of coating gold NPs with lipids, synthesized the gold nanoparticles inside pre-made liposomes.

\section{Nanotag targeting for in vivo imaging}

Raman imaging using SE(R)RS nanostars offers highly sensitive and specific disease detection. Their narrow, fingerprint-like spectra make $S E(R) R S$ nanotags ideally suited for in vivo multiplexed molecular imaging using up to 10 different 'flavors' of SERS nanoparticles [178]. Since the Raman reporters can be selected to generate a unique Raman fingerprint that is not found anywhere else in the biological system, unprecedented signal to background ratios can be obtained.

The Kircher group demonstrated that due to the femtomolar detection sensitivity of SE(R)RS nanostars, it was possible to image bulk tumor, microscopic infiltration into normal surrounding tissues, residual tumor after surgical resection, and 
even micrometastases in animal models that closely recapitulate pancreatic, prostate, breast, and colon carcinogenesis, among others, after intravenous administration [26, 132]. Non-targeted nanoparticles typically accumulate in tumor tissue via leaky neovasculature and are retained due to reduced clearance by a locally dysfunctional lymphatic system as well as increased influx of phagocytic cells - a phenomenon collectively known as the enhanced permeability and retention (EPR) effect [179-181].

While non-targeted nanoparticles have been shown to be highly effective in delineating solid tumors irrespective of their site of origin, no information is provided on the (molecular) composition of the tumor. To address this, targeted approaches using SE(R)RS nanoparticles were explored. The first demonstration of targeted SERS nanoparticles was performed by Nie and coworkers in 2008 who used thiol-PEG stabilized SERS nanoparticles that were linked to epidermal growth factor receptor (EGFR) targeting antibodies [101]. They showed that EGFR-targeting improved the tumor uptake of the SERS nanotags by 10 -fold relative to their non-targeted counterparts. Using a silica-coated gold nanostar core, Nayak et al. showed that tissue factor-targeted SERRS nanotags could visualize breast cancer metastases in the lungs, while non-targeted nanotags failed to do so under the same experimental conditions [182]. Similarly, Oseledchyk et al. created a cocktail of folate receptor-targeted and non-targeted SERS nanostars that could visualize folate receptor-expressing ovarian cancer metastases using a ratiometric imaging approach [106]. In another application, SERS nanostars were used for simultaneous detection of EGFR and programmed death-ligand 1 (PD-L1) in breast cancers using multiplexed detection of EGFR- and PD-L1-targeting antibody-labeled nanotags, respectively [183]. In a follow-up study, the same group utilized a combination of PD-L1- and CD8-targeted SERRS nanostars to that accurately detect PD-L1 ${ }^{+}$tumor cells and $\mathrm{CD}^{+} \mathrm{T}$ cells simultaneously in vivo. Moreover, this combination allowed the monitoring of treatment response, i.e. CD8 $\mathrm{T}$ cell recruitment to the tumor, following PD-L1 immunotherapy [184]. Lastly, Wang et al. used a cocktail of 3 different SERS nanostars. Their Raman reporters consisted of bioorthogonal molecules that embedded a combination of alkyne, azido, and cyano- functionality to generate a Raman fingerprint in the Raman silent region [185] - the spectral window between 1,750-2,750 where biological molecules typically do not emit any Raman signal. The reporters for the Raman silent region were subsequently encapsulated in silica, and targeting moieties were conjugated to the nanoparticle surface via heterobifunctional PEG linkers. The targeting moieties consisted of a nucleolin-targeting aptamer AS1411, an integrin $\alpha_{v} \beta_{3}$-targeting cyclic RGD peptide, or a CD44 antibody. Intravenous injection of this cocktail of targeted SERS nanostars allowed for multiplex phenotyping of breast cancer in animals bearing two MDA-MB-231 and MCF-7 tumors. Furthermore, this group confirmed that targeting improved tumor uptake 10-fold. In other studies, receptor-targeted SERS nanostars (e.g. integrin $\alpha_{\mathrm{v}} \beta_{3}$ and prostate-specific membrane antigen (PSMA)) enabled super-resolution SERS imaging to probe membrane receptor interactions in cells [186, 187], providing chemical information and spatial resolution with potential for diverse applications in life science and biomedicine such as for instance lateral flow assays for early (multiplexed) disease marker detection in bodily fluids [188-190].

The aforementioned studies demonstrate how the high signal sensitivity and specificity provided by SE(R)RS nanostars enable highly precise cancer imaging as well in vivo molecular imaging to allow for immediate tumor phenotyping as well as tumor monitoring.

\section{Multimodal imaging and theranostic applications}

Owing to their high uptake by tumors and high signal specificity, $\mathrm{SE}(\mathrm{R}) \mathrm{RS}$ nanostars offer tremendous opportunities for cancer diagnostics and imaging [191, 192]. However, like any other optical imaging technology, tissue depth penetration is limited and therefore deep tissue- or whole-body imaging is not supported by conventional Raman imaging. To improve detection of SERS nanotags at tissue depths that go beyond conventional Raman imaging detection, spatially offset Raman spectroscopy (SORS) or surface-enhanced spatially offset resonance Raman spectroscopy (SESORRS) can be used [42]. We recently discussed this up-and-coming technology in-depth [193]. Another approach would be to combine Raman imaging with magnetic resonance imaging (MRI), positron emission tomography (PET), fluorescence imaging and computed tomography (CT). The nanoparticle surface can be modified to incorporate metal chelating functional groups to chelate, for example, gadolinium or manganese to enable MRI functionality, or radioisotopes to render the nanoparticle PET-active $[184,194]$. In the case of silica-encapsulated SE(R)RS nanotags, the silica itself has high affinity for oxophilic cations such as gadolinium (MRI) or zirconium-89 (PET) and obviates the need for introduction of chelating groups [108, 195]. Less oxophilic (radio)isotopes such as copper-64 can be sequestered via thiolation of the SERS 
nanotag's silica surface using 3-mercaptopropyltrimethoxysilane (MPTMS) to produce multimodal PET-active SERS nanoparticles [196]. SERS nanotags that have gold cores provide intrinsic contrast on CT and could be used in a whole-body imaging setting [197]. Moreover, due to strong near-infrared absorption gold nanostars are particularly well-suited as contrast agents for photoacoustic (PA) imaging and SERRS-active gold nanostars enabled dual-modal Raman/PA imaging of glioblastoma [107]. The photoacoustic effect is achieved through rapid absorption of pulsed laser energy by the gold nanostar and its conversion to heat which causes a transient thermoplastic expansion of the gold nanostar core, that generates an ultrasonic band that can be detected using an ultrasound receiver. If instead of a pulsed laser, a high-power $(>100 \mathrm{~mW})$ continuous laser is used that matches the LSPR of the gold nanostars, the heating is not transient and can be used to heat up the local microenvironment surrounding the gold nanostars in a process known as the photothermal effect. In turn, this can be used to destroy tumor cells in a procedure called photothermal therapy (PTT), applicable to various cancer cells. SERS nanostars were used by Liu et al. to completely ablate the tumor using PTT after only 20 minutes of irradiation with no signs of recurrence after a week [198]. Another example of complete tumor ablation using nanostars was demonstrated by Gao et al., but this time after 30 minutes of laser irradiation [199]. Moreover, SERS nanotags used for PTT can be loaded with anticancer drugs for chemotherapy [200, 201], light-activatable molecules for photodynamic therapy (PDT) [109, 159] or release toxic and generate reactive oxygen species [202, 203].

\section{Conclusions and outlook}

In recent years, SERS nanotags have evolved from rather simple nanoparticles used primarily for in vitro assays to complex multicomponent nanoconstructs tailored for use in biomedical diagnostic and therapeutic applications. Even though the principles of the design of SERS nanotags have already been outlined, the development of every aspect of a SERS nanotag is a subject of ongoing research and improvement.

Significant progress has been made in diversifying anisotropic metal core shapes and their respective synthesis protocols, allowing researchers to finely tune the final shape, size and surface properties of nanostars and reach enhancement factors unattainable with spherical nanoparticles. However, preparation of narrowly dispersed nanoparticle preparations remains a challenge and, consequently, there is a need for reproducible synthesis procedures to obtain nanoparticles with structural homogeneity with pristine surfaces; continuous in-line microfluidics-based synthesis approaches could provide a potential solution. While the general design principles of Raman reporter molecules can be considered established (i.e. high Raman cross-section, resonance with laser, high affinity for plasmonic core surface), to date only a limited amount of dye-scaffolds has been used and the most widely-used resonant Raman reporters are based on commercial cyanine dyes. Development of novel dye designs would greatly benefit the multiplex capabilities of SE(R)RS-based biomedical strategies. As discussed, SERS nanotag coating is the most diverse component of the construct as it not only is involved in nanoparticle stabilization, but also acts as a base for targeting moiety conjugation, as well as (intrinsic) chelation of medical isotopes to produce targeted-SERS nanotags with multimodal capabilities.

Preclinical evaluation of gold nanostar-based SERS nanotags have shown the tremendous promise of such agents for highly sensitive disease (marker) detection and even therapeutic intervention using PTT. However, upon intravenous administration, SERS nanotags, like any nanoparticle of similar size or composition, not only accumulate in tumor tissues, but also in organs of the reticuloendothelial system (RES) such as liver and spleen. Although gold is metabolically inert and silica-based coatings are fully biodegradable, the effect of long-term retention on these organs remains unclear. Therefore, efforts are being directed to designing truly biodegradable or excretable SERS nanotags that retain their high signal intensity.

Lastly, design of medical-grade Raman imaging systems are required for the clinical translation of contrast-enhanced Raman imaging approaches. We recently demonstrated for the first time that a clinical Raman endoscope [204] enabled the detection of incipient cancers in the gastrointestinal tract following SERRS nanoparticle administration in preclinical models of colorectal carcinogenesis [132]. Similarly, a wide-field Raman imaging system could enable Raman-guided tumor resection in cancer patients. While such systems are currently being developed [205], they currently do not enable real-time imaging due to the relatively long acquisition times/area relative to fluorescence imaging. However, rapid advancements in optical technologies and new insights in Raman instrument design may someday lead to the development of a real-time, wide-field Raman imaging system that will enable Raman-guided macroscopic tumor resection and photothermal ablation of residual microscopic tumor cells in the resection bed when paired with 
intravenously administered SERRS nanostars; a truly nanotheranostic platform.

\section{Acknowledgements}

The following funding sources (MFK) are acknowledged: NIH R01 EB017748, R01 CA222836, and an Innovation Research Fund from the Dana-Farber Cancer Institute. FN acknowledges the Trustee Science Committee Postdoctoral Fellowship from the Dana-Farber Cancer Institute.

\section{Competing Interests}

The authors have declared that no competing interest exists.

\section{References}

1. Raman CV, Krishnan KS. A New Type of Secondary Radiation. Nature. 1928; 121: 501-2.

2. Smekal A. Zur Quantentheorie der Dispersion. Naturwissenschaften. 1923; 11: 873-5.

3. Smith E, Dent G. Modern Raman Spectroscopy - A Practical Approach: John Wiley \& Sons, Ltd; 2005.

4. Fleischmann M, Hendra PJ, McQuillan AJ. Raman spectra of pyridine adsorbed at a silver electrode. Chemical Physics Letters. 1974; 26: 163-6.

5. Moskovits M. Surface-enhanced spectroscopy. Reviews of Modern Physics. 1985; 57: 783-826.

6. Le Ru EC, Blackie E, Meyer M, Etchegoin PG. Surface enhanced Raman scattering enhancement factors: a comprehensive study. Journal of Physical Chemistry C. 2007; 111: 13794-803.

7. Kleinman SL, Frontiera RR, Henry AI, Dieringer JA, Van Duyne RP. Creating, characterizing, and controlling chemistry with SERS hot spots. Phys Chem Chem Phys. 2013; 15: 21-36.

8. McNay G, Eustace D, Smith WE, Faulds K, Graham D. Surface-enhanced Raman scattering (SERS) and surface-enhanced resonance Raman scattering (SERRS): a review of applications. Appl Spectrosc. 2011; 65: 825-37.

9. Cardinal MF, Vander Ende E, Hackler RA, McAnally MO, Stair PC, Schatz GC, et al. Expanding applications of SERS through versatile nanomaterials engineering. Chem Soc Rev. 2017; 46: 3886-903.

10. Langer J, Jimenez de Aberasturi D, Aizpurua J, Alvarez-Puebla RA, Auguie B, Baumberg JJ, et al. Present and Future of Surface-Enhanced Raman Scattering. ACS Nano. 2020; 14: 28-117.

11. Lane LA, Qian X, Nie S. SERS Nanoparticles in Medicine: From Label-Free Detection to Spectroscopic Tagging. Chem Rev. 2015; 115: 10489-529.

12. Harmsen S, Bedics MA, Wall MA, Huang R, Detty MR, Kircher MF Rational design of a chalcogenopyrylium-based surface-enhanced resonance Raman scattering nanoprobe with attomolar sensitivity. Nat Commun. 2015; 6: 6570.

13. Harmsen S, Wall MA, Huang R, Kircher MF. Cancer imaging using surface-enhanced resonance Raman scattering nanoparticles. Nat Protoc. 2017; 12: 1400-14.

14. Stiles PL, Dieringer JA, Shah NC, Van Duyne RP. Surface-enhanced Raman spectroscopy. Annu Rev Anal Chem (Palo Alto Calif). 2008; 1: 601-26.

15. Schlucker S. Surface-enhanced Raman spectroscopy: concepts and chemical applications. Angew Chem Int Ed Engl. 2014; 53: 4756-95.

16. Li M, Cushing SK, Zhang J, Lankford J, Aguilar ZP, Ma D, et al. Shape-dependent surface-enhanced Raman scattering in gold-Ramanprobe-silica sandwiched nanoparticles for biocompatible applications. Nanotechnology. 2012; 23

17. Pazos-Perez N, Guerrini L, Alvarez-Puebla RA. Plasmon Tunability of Gold Nanostars at the Tip Apexes. ACS Omega. 2018; 3: 17173-9.

18. Chase B, Parkinson B. A study of the wavelength and potential dependence of surface enhanced Raman scattering on copper, silver, and gold electrodes. The Journal of Physical Chemistry. 1991; 95: 7810-3.

19. Sengul AB, Asmatulu E. Toxicity of metal and metal oxide nanoparticles: a review. Environmental Chemistry Letters. 2020; 18: 1659-83.

20. Sukhanova A, Bozrova S, Sokolov P, Berestovoy M, Karaulov A, Nabiev I. Dependence of Nanoparticle Toxicity on Their Physical and Chemical Properties. Nanoscale Res Lett. 2018; 13: 21.
21. Smith AM, Mancini MC, Nie S. Bioimaging: second window for in vivo imaging. Nat Nanotechnol. 2009; 4: 710-1.

22. Link S, El-Sayed MA. Size and temperature dependence of the plasmon absorption of colloidal gold nanoparticles. Journal of Physical Chemistry B. 1999; 103: 4212-7.

23. Park JE, Lee Y, Nam JM. Precisely Shaped, Uniformly Formed Gold Nanocubes with Ultrahigh Reproducibility in Single-Particle Scattering and Surface-Enhanced Raman Scattering. Nano Lett. 2018; 18: 6475-82.

24. Nikoobakht B, El-Sayed MA. Surface-enhanced Raman scattering studies on aggregated gold nanorods. Journal of Physical Chemistry A. 2003; 107: 3372-8

25. Reguera J, Langer J, Jimenez de Aberasturi D, Liz-Marzan LM. Anisotropic metal nanoparticles for surface enhanced Raman scattering. Chemical Society Reviews. 2017; 46: 3866-85.

26. Harmsen S, Huang R, Wall MA, Karabeber H, Samii JM, Spaliviero M, et al. Surface-enhanced resonance Raman scattering nanostars for high-precision cancer imaging. Science Translational Medicine. 2015; 7.

27. Xie J, Lee JY, Wang DIC. Seedless, surfactantless, high-yield synthesis of branched gold nanocrystals in HEPES buffer solution. Chemistry of Materials. 2007; 19: 2823-30.

28. Grzelczak M, Perez-Juste J, Mulvaney P, Liz-Marzan LM. Shape control in gold nanoparticle synthesis. Chemical Society Reviews. 2008; 37: 1783-91.

29. Chandra K, Kumar V, Werner SE, Odom TW. Separation of Stabilized MOPS Gold Nanostars by Density Gradient Centrifugation. ACS Omega. 2017; 2: 4878-84.

30. Sau TK, Murphy CJ. Room temperature, high-yield synthesis of multiple shapes of gold nanoparticles in aqueous solution. Journal of the American Chemical Society. 2004; 126: 8648-9.

31. Alkilany AM, Nagaria PK, Hexel CR, Shaw TJ, Murphy CJ, Wyatt MD. Cellular Uptake and Cytotoxicity of Gold Nanorods: Molecular Origin of Cytotoxicity and Surface Effects. Small. 2009; 5: 701-8.

32. Kuo $\mathrm{CH}$, Huang $\mathrm{MH}$. Synthesis of branched gold nanocrystals by a seeding growth approach. Langmuir. 2005; 21: 2012-6.

33. Sasidharan S, Bahadur D, Srivastava R. Rapid, One-Pot, Protein-Mediated Green Synthesis of Gold Nanostars for Computed Tomographic Imaging and Photothermal Therapy of Cancer. Acs Sustainable Chemistry \& Engineering. 2017; 5: 10163-75.

34. Liebig F, Henning R, Sarhan RM, Prietzel C, Schmitt CNZ, Bargheer M, et al. A simple one-step procedure to synthesise gold nanostars in concentrated aqueous surfactant solutions. Rsc Advances. 2019; 9: 23633-41.

35. Li Y, Ma J, Ma Z. Synthesis of gold nanostars with tunable morphology and their electrochemical application for hydrogen peroxide sensing. Electrochimica Acta. 2013; 108: 435-40.

36. Pallavicini P, Dona A, Casu A, Chirico G, Collini M, Dacarro G, et al. Triton X-100 for three-plasmon gold nanostars with two photothermally active NIR (near IR) and SWIR (short-wavelength IR) channels. Chemical Communications. 2013; 49: 6265-7.

37. Yuan H, Khoury CG, Hwang H, Wilson CM, Grant GA, Tuan V-D. Gold nanostars: surfactant-free synthesis, 3D modelling, and two-photon photoluminescence imaging. Nanotechnology. 2012; 23.

38. Ramsey JD, Zhou L, Almlie CK, Lange JD, Burrows SM. Achieving plasmon reproducibility from surfactant free gold nanostar synthesis. New Journal of Chemistry. 2015; 39: 9098-108.

39. Indrasekara ASDS, Meyers $S$, Shubeita $S$, Feldman LC, Gustafsson T, Fabris L. Gold nanostar substrates for SERS-based chemical sensing in the femtomolar regime. Nanoscale. 2014; 6: 8891-9.

40. de Silva Indrasekara AS, Johnson SF, Odion RA, Tuan V-D. Manipulation of the Geometry and Modulation of the Optical Response of Surfactant-Free Gold Nanostars: A Systematic Bottom-Up Synthesis. Acs Omega. 2018; 3: 2202-10.

41. Andreou C, Oseledchyk A, Nicolson F, Berisha N, Pal S, Kircher MF. Surface-enhanced Resonance Raman Scattering Nanoprobe Ratiometry for Detecting Microscopic Ovarian Cancer via Folate Receptor Targeting. Jove-Journal of Visualized Experiments. 2019

42. Nicolson F, Andreiuk B, Andreou C, Hsu H-T, Rudder S, Kircher MF. Non-invasive In Vivo Imaging of Cancer Using Surface-Enhanced Spatially Offset Raman Spectroscopy (SESORS). Theranostics. 2019; 9: 5899-913.

43. Pérez-Juste J, Pastoriza-Santos I, Liz-Marzán LM, Mulvaney P. Gold nanorods: Synthesis, characterization and applications. Coordination Chemistry Reviews. 2005; 249: 1870-901.

44. Jana NR, Gearheart L, Murphy CJ. Seed-Mediated Growth Approach for Shape-Controlled Synthesis of Spheroidal and Rod-like Gold Nanoparticles Using a Surfactant Template. Advanced Materials. 2001; 13: 1389-93. 
45. Yuan H, Ma W, Chen C, Zhao J, Liu J, Zhu H, et al. Shape and SPR Evolution of Thorny Gold Nanoparticles Promoted by Silver Ions. Chemistry of Materials. 2007; 19: 1592-600.

46. Nehl CL, Liao H, Hafner JH. Optical Properties of Star-Shaped Gold Nanoparticles. Nano Letters. 2006; 6: 683-8.

47. Elechiguerra JL, Reyes-Gasga J, Yacaman MJ. The role of twinning in shape evolution of anisotropic noble metal nanostructures. Journal of Materials Chemistry. 2006; 16: 3906-19.

48. Sajanlal PR, Pradeep T. Mesoflowers: A new class of highly efficient surface-enhanced Raman active and infrared-absorbing materials. Nano Research. 2009; 2: 306-20.

49. Atta S, Beetz M, Fabris L. Understanding the role of AgNO3 concentration and seed morphology in the achievement of tunable shape control in gold nanostars. Nanoscale. 2019; 11: 2946-58.

50. Sánchez-Iglesias A, Winckelmans N, Altantzis T, Bals S, Grzelczak M, Liz-Marzán LM. High-Yield Seeded Growth of Monodisperse Pentatwinned Gold Nanoparticles through Thermally Induced Seed Twinning. Journal of the American Chemical Society. 2017; 139: 107-10.

51. Liu M, Guyot-Sionnest P. Mechanism of Silver(I)-Assisted Growth of Gold Nanorods and Bipyramids. The Journal of Physical Chemistry B. 2005; 109: 22192-200.

52. Cheng L-C, Huang J-H, Chen HM, Lai T-C, Yang K-Y, Liu R-S, et al. Seedless, silver-induced synthesis of star-shaped gold/silver bimetallic nanoparticles as high efficiency photothermal therapy reagent. Journal of Materials Chemistry. 2012; 22: 2244-53.

53. Sheen Mers SV, Umadevi S, Ganesh V. Controlled Growth of Gold Nanostars: Effect of Spike Length on SERS Signal Enhancement. ChemPhysChem. 2017; 18: 1358-69.

54. Personick ML, Langille MR, Zhang J, Mirkin CA. Shape Control of Gold Nanoparticles by Silver Underpotential Deposition. Nano Letters. 2011; 11: 3394-8.

55. Habib A, Tabata M, Wu YG. Formation of gold nanoparticles by Good's buffers. Bulletin of the Chemical Society of Japan. 2005; 78: 262-9.

56. Chandra K, Culver KSB, Werner SE, Lee RC, Odom TW. Manipulating the Anisotropic Structure of Gold Nanostars using Good's Buffers. Chemistry of Materials. 2016; 28: 6763-9.

57. Pallares RM, Stilson T, Choo P, Hu J, Odom TW. Using Good's Buffers To Control the Anisotropic Structure and Optical Properties of Spiky Gold Nanoparticles for Refractive Index Sensing. Acs Applied Nano Materials. 2019; 2: 5266-71.

58. Maiorano G, Rizzello L, Malvindi MA, Shankar SS, Martiradonna L, Falqui A, et al. Monodispersed and size-controlled multibranched gold nanoparticles with nanoscale tuning of surface morphology. Nanoscale. 2011; 3: 2227-32.

59. Webb JA, Erwin WR, Zarick HF, Aufrecht J, Manning HW, Lang MJ, et al. Geometry-Dependent Plasmonic Tunability and Photothermal Characteristics of Multibranched Gold Nanoantennas. Journal of Physical Chemistry C. 2014; 118: 3696-707.

60. Mulder DW, Phiri MM, Jordaan A, Vorster BC. Modified HEPES one-pot synthetic strategy for gold nanostars. Royal Society Open Science. 2019; 6.

61. Xi W, Haes AJ. Elucidation of HEPES Affinity to and Structure on Gold Nanostars. Journal of the American Chemical Society. 2019; 141: 4034-42.

62. Chandra K, Rugg BK, Ratner MA, Wasielewski MR, Odom TW. Detecting and Visualizing Reaction Intermediates of Anisotropic Nanoparticle Growth. Journal of the American Chemical Society. 2018; 140: 3219-22.

63. Senthil Kumar P, Pastoriza-Santos I, Rodriguez-Gonzalez B, Garcia de Abajo FJ, Liz-Marzan LM. High-yield synthesis and optical response of gold nanostars. Nanotechnology. 2008; 19.

64. Barbosa S, Agrawal A, Rodriguez-Lorenzo L, Pastoriza-Santos I, Alvarez-Puebla RA, Kornowski A, et al. Tuning Size and Sensing Properties in Colloidal Gold Nanostars. Langmuir. 2010; 26: 14943-50.

65. Khoury CG, Vo-Dinh T. Gold Nanostars For Surface-Enhanced Raman Scattering: Synthesis, Characterization and Optimization. Journal of Physical Chemistry C. 2008; 112: 18849-59.

66. Niu W, Chua YAA, Zhang W, Huang H, Lu X. Highly Symmetric Gold Nanostars: Crystallographic Control and Surface-Enhanced Raman Scattering Property. Journal of the American Chemical Society. 2015; 137: 10460-3.

67. Jana D, Matti C, He J, Sagle L. Capping Agent-Free Gold Nanostars Show Greatly Increased Versatility and Sensitivity for Biosensing. Analytical Chemistry. 2015; 87: 3964-72.

68. Li J, Wu J, Zhang X, Liu Y, Zhou D, Sun H, et al. Controllable Synthesis of Stable Urchin-like Gold Nanoparticles Using Hydroquinone to Tune the Reactivity of Gold Chloride. Journal of Physical Chemistry C. 2011; 115: 3630-7.

69. Schuetz M, Steinigeweg D, Salehi M, Koempe K, Schluecker S. Hydrophilically stabilized gold nanostars as SERS labels for tissue imaging of the tumor suppressor p63 by immuno-SERS microscopy. Chemical Communications. 2011; 47: 4216-8.

70. Moukarzel W, Fitremann J, Marty J-D. Seed-less amino-sugar mediated synthesis of gold nanostars. Nanoscale. 2011; 3: 3285-90.

71. Zhang X-L, Zheng C, Zhang Y, Yang H-H, Liu X, Liu J. One-pot synthesis of gold nanostars using plant polyphenols for cancer photoacoustic imaging and photothermal therapy. Journal of Nanoparticle Research. $2016 ; 18$.

72. Wall MA, Harmsen S, Pal S, Zhang L, Arianna G, Lombardi JR, et al. Surfactant-Free Shape Control of Gold Nanoparticles Enabled by Unified Theoretical Framework of Nanocrystal Synthesis. Advanced Materials. $2017 ; 29$.

73. Cao X, Chen S, Li W, Li J, Bi L, Shi H. One-step synthesis of highly-branched gold nanostructures and its application in fabrication of SERS-active substrates. Aip Advances. 2018; 8.

74. Sajitha M, Vindhyasarumi A, Gopi A, Yoosaf K. Shape controlled synthesis of multi-branched gold nanocrystals through a facile one-pot bifunctional biomolecular approach. Rsc Advances. 2015; 5: 98318-24.

75. Bian P, Yuan J, Han H, Ma Z. A Seedless, Surfactant-Free Synthesis of Gold Nanospheres, Nanosheets, and Nanostars Based on Functionalized Tryptophan. Journal of Nanoscience and Nanotechnology. 2016; 16: 7503-8

76. Yang D-P, Liu X, Teng CP, Owh C, Win KY, Lin M, et al. Unexpected formation of gold nanoflowers by a green synthesis method as agents for a safe and effective photothermal therapy. Nanoscale. 2017; 9: 15753-9.

77. Valencia PM, Farokhzad OC, Karnik R, Langer R. Microfluidic technologies for accelerating the clinical translation of nanoparticles. Nature Nanotechnology. 2012; 7: 623-9.

78. Ma J, Lee SM-Y, Yi C, Li C-W. Controllable synthesis of functional nanoparticles by microfluidic platforms for biomedical applications - a review. Lab on a Chip. 2017; 17: 209-26.

79. Liu Z, Fontana F, Python A, Hirvonen JT, Santos HA. Microfluidics for Production of Particles: Mechanism, Methodology, and Applications. Small. 2020; 16: 1904673.

80. Nette J, Howes PD, deMello AJ. Microfluidic Synthesis of Luminescent and Plasmonic Nanoparticles: Fast, Efficient, and Data-Rich. Advanced Materials Technologies. 2020; n/a: 2000060.

81. Sebastián V, Jensen KF. Nanoengineering a library of metallic nanostructures using a single microfluidic reactor. Nanoscale. 2016; 8: 15288-95

82. Shang L, Cheng Y, Zhao Y. Emerging Droplet Microfluidics. Chemical Reviews. 2017; 117: 7964-8040.

83. Suea-Ngam A, Howes PD, Srisa-Art M, deMello AJ. Droplet microfluidics: from proof-of-concept to real-world utility? Chemical Communications. 2019; 55: 9895-903.

84. Silvestri A, Lay L, Psaro R, Polito L, Evangelisti C. Fluidic Manufacture of Star-Shaped Gold Nanoparticles. Chemistry - A European Journal. 2017; 23: 9732-5.

85. Abalde-Cela S, Taladriz-Blanco P, de Oliveira MG, Abell C. Droplet microfluidics for the highly controlled synthesis of branched gold nanoparticles. Scientific Reports. 2018; 8: 2440.

86. Langer J, de Aberasturi DJ, Aizpurua J, Alvarez-Puebla RA, Auguie B, Baumberg JJ, et al. Present and Future of Surface-Enhanced Raman Scattering. Acs Nano. 2020; 14: 28-117.

87. Wang Y, Schluecker S. Rational design and synthesis of SERS labels. Analyst. 2013; 138: 2224-38.

88. Shan B, Pu Y, Chen Y, Liao M, Li M. Novel SERS labels: Rational design, functional integration and biomedical applications. Coordination Chemistry Reviews. 2018; 371: 11-37.

89. Laing S, Jamieson LE, Faulds K, Graham D. Surface-enhanced Raman spectroscopy for in vivo biosensing. Nature Reviews Chemistry. 2017; 1.

90. Hakkinen $\mathrm{H}$. The gold-sulfur interface at the nanoscale. Nature Chemistry. 2012; 4: 443-55.

91. Love JC, Estroff LA, Kriebel JK, Nuzzo RG, Whitesides GM. Self-assembled monolayers of thiolates on metals as a form of nanotechnology. Chemical Reviews. 2005; 105: 1103-69.

92. Wang G, Lipert RJ, Jain M, Kaur S, Chakraboty S, Torres MP, et al. Detection of the Potential Pancreatic Cancer Marker MUC4 in Serum Using Surface-Enhanced Raman Scattering. Analytical Chemistry. 2011; 83: 2554-61.

93. Xu L, Yan W, Ma W, Kuang H, Wu X, Liu L, et al. SERS Encoded Silver Pyramids for Attomolar Detection of Multiplexed Disease Biomarkers. Advanced Materials. 2015; 27: 1706-+

94. Hu F, Zhang Y, Chen G, Li C, Wang Q. Double-Walled Au Nanocage/SiO2 Nanorattles: Integrating SERS Imaging, Drug Delivery and Photothermal Therapy. Small. 2015; 11: 985-93.

95. Orendorff CI, Gole A, Sau TK, Murphy CJ. Surface-enhanced Raman spectroscopy of self-assembled monolayers: Sandwich architecture and nanoparticle shape dependence. Analytical Chemistry. 2005; 77: 3261-6. 
96. Schuetz M, Mueller CI, Salehi M, Lambert C, Schluecker S. Design and synthesis of Raman reporter molecules for tissue imaging by immuno-SERS microscopy. Journal of Biophotonics. 2011; 4: 453-63.

97. Zhang L, Zhang R, Gao M, Zhang X. Facile synthesis of thiol and alkynyl contained SERS reporter molecular and its usage in assembly of polydopamine protected bioorthogonal SERS tag for live cell imaging. Talanta. 2016; 158: 315-21.

98. Grubisha DS, Lipert RJ, Park HY, Driskell J, Porter MD. Femtomolar detection of prostate-specific antigen: An immunoassay based on surface-enhanced Raman scattering and immunogold labels. Analytical Chemistry. 2003; 75: 5936-43.

99. Nie SM, Emery SR. Probing single molecules and single nanoparticles by surface-enhanced Raman scattering. Science. 1997; 275: 1102-6.

100. Hildebrandt P, Stockburger M. SURFACE-ENHANCED RESONANCE RAMAN-SPECTROSCOPY OF RHODAMINE-6G ADSORBED ON COLLOIDAL SILVER. Journal of Physical Chemistry. 1984; 88: 5935-44.

101. Qian X, Peng X-H, Ansari DO, Yin-Goen Q, Chen GZ, Shin DM, et al. In vivo tumor targeting and spectroscopic detection with surface-enhanced Raman nanoparticle tags. Nature Biotechnology. 2008; 26: 83-90.

102. Li M, Cushing SK, Zhang J, Suri S, Evans R, Petros WP, et al. Three-Dimensional Hierarchical Plasmonic Nano-Architecture Enhanced Surface-Enhanced Raman Scattering Immunosensor for Cancer Biomarker Detection in Blood Plasma. Acs Nano. 2013; 7: 4967-76.

103. von Maltzahn G, Centrone A, Park J-H, Ramanathan R, Sailor MJ, Hatton TA, et al. SERS-Coded Gold Nanorods as a Multifunctional Platform for Densely Multiplexed Near-infrared Imaging and Photothermal Heating. Advanced Materials. 2009; 21: 3175-+.

104. Yuan H, Liu Y, Fales AM, Li YL, Liu J, Vo-Dinh T. Quantitative Surface-Enhanced Resonant Raman Scattering Multiplexing of Biocompatible Gold Nanostars for in Vitro and ex Vivo Detection. Analytical Chemistry. 2013; 85: 208-12.

105. Pal S, Harmsen S, Oseledchyk A, Hsu H-T, Kircher MF. MUC1 Aptamer Targeted SERS Nanoprobes. Advanced Functional Materials. 2017; 27.

106. Oseledchyk A, Andreou C, Wall MA, Kircher MF. Folate-Targeted Surface-Enhanced Resonance Raman Scattering Nanoprobe Ratiometry for Detection of Microscopic Ovarian Cancer. Acs Nano. 2017; 11: 1488-97.

107. Neuschmelting V, Harmsen S, Beziere N, Lockau H, Hsu H-T, Huang R, et al. Dual-Modality Surface-Enhanced Resonance Raman Scattering and Multispectral Optoacoustic Tomography Nanoparticle Approach for Brain Tumor Delineation. Small. 2018; 14.

108. Wall MA, Shaffer TM, Harmsen S, Tschaharganeh D-F, Huang C-H, Lowe SW, et al. Chelator-Free Radiolabeling of SERRS Nanoparticles for Whole-Body PET and Intraoperative Raman Imaging. Theranostics. 2017; 7: 3068-77.

109. Nagy-Simon T, Potara M, Craciun AM, Licarete E, Astilean S. IR780-dye loaded gold nanoparticles as new near infrared activatable nanotheranostic agents for simultaneous photodynamic and photothermal therapy and intracellular tracking by surface enhanced resonant Raman scattering imaging. Journal of Colloid and Interface Science. 2018; 517: 239-50

110. Mohs AM, Mancini MC, Singhal S, Provenzale JM, Leyland-Jones B, Wang MD, et al. Hand-held Spectroscopic Device for In Vivo and Intraoperative Tumor Detection: Contrast Enhancement, Detection Sensitivity, and Tissue Penetration. Analytical Chemistry. 2010; 82: 9058-65.

111. McLintock A, Cunha-Matos CA, Zagnoni M, Millington OR, Wark AW. Universal Surface-Enhanced Raman Tags: Individual Nanorods for Measurements from the Visible to the Infrared (514-1064 nm). Acs Nano. 2014; 8: 8600-9.

112. Samanta A, Maiti KK, Soh K-S, Liao X, Vendrell M, Dinish US, et al. Ultrasensitive Near-Infrared Raman Reporters for SERS-Based In Vivo Cancer Detection. Angewandte Chemie-International Edition. 2011; 50: 6089-92.

113. Amendola V, Meneghetti M. Exploring How to Increase the Brightness of Surface-Enhanced Raman Spectroscopy Nanolabels: The Effect of the Raman-Active Molecules and of the Label Size. Advanced Functional Materials. 2012; 22: 353-60.

114. Pal S, Ray A, Andreou C, Zhou Y, Rakshit T, Wlodarczyk M, et al. DNA-enabled rational design of fluorescence-Raman bimodal nanoprobes for cancer imaging and therapy. Nature Communications. $2019 ; 10$.

115. Guehlke M, Heiner Z, Kneipp J. Surface-Enhanced Raman and Surface-Enhanced Hyper-Raman Scattering of Thiol-Functionalized Carotene. Journal of Physical Chemistry C. 2016; 120: 20702-9.

116. Keller T, Brem S, Tran V, Sritharan O, Schaefer D, Schluecker S. Rational design of thiolated polyenes as trifunctional Raman reporter molecules in surface-enhanced Raman scattering nanotags for cytokine detection in a lateral flow assay. Journal of Biophotonics. 2020.

117. Brem S, Schluecker S. Surface-Enhanced Raman Spectroscopy and Density Functional Theory Calculations of a Rationally Designed Rhodamine with Thiol Groups at the Xanthene Ring. Journal of Physical Chemistry C. 2017; 121: 15310-7.

118. Maiti KK, Dinish US, Fu CY, Lee J-J, Soh K-S, Yun S-W, et al. Development of biocompatible SERS nanotag with increased stability by chemisorption of reporter molecule for in vivo cancer detection. Biosensors \& Bioelectronics. 2010; 26: 398-403.

119. Dinish US, Song Z, Ho CJH, Balasundaram G, Attia ABE, Lu X, et al. Single Molecule with Dual Function on Nanogold: Biofunctionalized Construct for In Vivo Photoacoustic Imaging and SERS Biosensing. Advanced Functional Materials. 2015; 25: 2316-25.

120. Narayanan N, Karunakaran V, Paul W, Venugopal K, Sujathan K, Maiti KK. Aggregation induced Raman scattering of squaraine dye: Implementation in diagnosis of cervical cancer dysplasia by SERS imaging. Biosensors \& Bioelectronics. 2015; 70: 145-52

121. Ramya AN, Joseph MM, Nair JB, Karunakaran V, Narayanan N, Maiti KK. New Insight of Tetraphenylethylene-based Raman Signatures for Targeted SERS Nanoprobe Construction Toward Prostate Cancer Cell Detection. Acs Applied Materials \& Interfaces. 2016; 8: 10220-5.

122. Adarsh N, Ramya AN, Maiti KK, Ramaiah D. Unveiling NIR Aza-Boron-Dipyrromethene (BODIPY) Dyes as Raman Probes: Surface-Enhanced Raman Scattering (SERS)-Guided Selective Detection and Imaging of Human Cancer Cells. Chemistry-a European Journal. 2017; 23: 14286-91.

123. Zhang W, Liu J, Niu W, Yan H, Lu X, Liu B. Tip-Selective Growth of Silver on Gold Nanostars for Surface-Enhanced Raman Scattering. Acs Applied Materials \& Interfaces. 2018; 10: 14850-6.

124. Yue Q, Gao X, Yu Y, Li Y, Hua W, Fan K, et al. An EGFRvIII targeted dual-modal gold nanoprobe for imaging-guided brain tumor surgery. Nanoscale. 2017; 9: 7930-40.

125. Nicolson F, Jamieson LE, Mabbott S, Plakas K, Shand NC, Detty MR, et al. Through tissue imaging of a live breast cancer tumour model using handheld surface enhanced spatially offset resonance Raman spectroscopy (SESORRS). Chemical Science. 2018; 9: 3788-92.

126. Kearns H, Ali F, Bedics MA, Shand NC, Faulds K, Detty MR, et al. Sensitive SERS nanotags for use with a hand-held $1064 \mathrm{~nm}$ Raman spectrometer. Royal Society Open Science. 2017; 4.

127. Harmsen S, Bedics MA, Wall MA, Huang R, Detty MR, Kircher MF. Rational design of a chalcogenopyrylium-based surface-enhanced resonance Raman scattering nanoprobe with attomolar sensitivity. Nature Communications. 2015; 6.

128. Kang H, Buchman JT, Rodriguez RS, Ring HL, He J, Bantz KC, et al. Stabilization of Silver and Gold Nanoparticles: Preservation and Improvement of Plasmonic Functionalities. Chemical Reviews. 2019; 119: 664-99.

129. Jokerst JV, Lobovkina T, Zare RN, Gambhir SS. Nanoparticle PEGylation for imaging and therapy. Nanomedicine. 2011; 6: 715-28.

130. Suk JS, Xu Q, Kim N, Hanes J, Ensign LM. PEGylation as a strategy for improving nanoparticle-based drug and gene delivery. Advanced Drug Delivery Reviews. 2016; 99: 28-51.

131. Feng J, Chen L, Xia Y, Xing J, Li Z, Qian Q, et al. Bioconjugation of Gold Nanobipyramids for SERS Detection and Targeted Photothermal Therapy in Breast Cancer. Acs Biomaterials Science \& Engineering. 2017; 3: 608-18

132. Harmsen S, Rogalla S, Huang R, Spaliviero M, Neuschmelting V, Hayakawa Y, et al. Detection of Premalignant Gastrointestinal Lesions Using Surface-Enhanced Resonance Raman Scattering-Nanoparticle Endoscopy. Acs Nano. 2019; 13: 1354-64.

133. Fales AM, Crawford BM, Vo-Dinh T. Folate Receptor-Targeted Theranostic Nanoconstruct for Surface-Enhanced Raman Scattering Imaging and Photodynamic Therapy. Acs Omega. 2016; 1: 730-5.

134. Liu YL, Ai KL, Lu LH. Polydopamine and Its Derivative Materials: Synthesis and Promising Applications in Energy, Environmental, and Biomedical Fields. Chemical Reviews. 2014; 114: 5057-115.

135. Liu XS, Cao JM, Li H, Li JY, Jin Q, Ren KF, et al. Mussel-Inspired Polydopamine: A Biocompatible and Ultrastable Coating for Nanoparticles in Vivo. Acs Nano. 2013; 7: 9384-95.

136. Jiang $\mathrm{CH}$, Wang $\mathrm{Y}$, Wang JW, Song W, Lu LH. Achieving ultrasensitive in vivo detection of bone crack with polydopamine-capsulated surface-enhanced Raman nanoparticle. Biomaterials. 2017; 114: 54-61.

137. Zhang F, Lees E, Amin F, Gil PR, Yang F, Mulvaney P, et al. Polymer-Coated Nanoparticles: A Universal Tool for Biolabelling Experiments. Small. 2011; 7: 3113-27.

138. Jimenez de Aberasturi D, Serrano-Montes AB, Langer J, Henriksen-Lacey M, Parak WJ, Liz-Marzan LM. Surface Enhanced 
Raman Scattering Encoded Gold Nanostars for Multiplexed Cell Discrimination. Chemistry of Materials. 2016; 28: 6779-90.

139. Yang MX, Chen T, Lau WS, Wang Y, Tang QH, Yang YH, et al. Development of Polymer-Encapsulated Metal Nanoparticles as Surface-Enhanced Raman Scattering Probes (vol 5, pg 198, 2009). Small. 2011; 7: 2412-.

140. Song J, Zhou J, Duan H. Self-Assembled Plasmonic Vesicles of SERS-Encoded Amphiphilic Gold Nanoparticles for Cancer Cell Targeting and Traceable Intracellular Drug Delivery. Journal of the American Chemical Society. 2012; 134: 13458-69.

141. Gao X, Yue Q, Liu Z, Ke M, Zhou X, Li S, et al. Guiding Brain-Tumor Surgery via Blood-Brain-Barrier-Permeable Gold Nanoprobes with Acid-Triggered MRI/SERRS Signals. Advanced Materials. 2017; 29.

142. Liu ZM, Ye BG, Jin M, Chen HL, Zhong HQ, Wang XP, et al. Dye-free near-infrared surface-enhanced Raman scattering nanoprobes for bioimaging and high-performance photothermal cancer therapy. Nanoscale. 2015; 7: 6754-61.

143. Iacono P, Karabeber H, Kircher MF. A "Schizophotonic" All-In-One Nanoparticle Coating for Multiplexed SE(R)RS Biomedical Imaging. Angewandte Chemie-International Edition. 2014; 53: 11756-61.

144. Bodelon G, Montes-Garcia V, Fernandez-Lopez C, Pastoriza-Santos I, Perez-Juste J, Liz-Marzan LM. Au@pNIPAM SERRS Tags for Multiplex Immunophenotyping Cellular Receptors and Imaging Tumor Cells. Small. 2015; 11: 4149-57.

145. Kearns H, Shand NC, Faulds K, Graham D. Laser induced SERS switching using plasmonic heating of PNIPAM coated HGNs. Chemical Communications. 2015; 51: 8138-41.

146. Song J, Yang X, Yang Z, Lin L, Liu Y, Zhou Z, et al. Rational Design of Branched Nanoporous Gold Nanoshells with Enhanced Physico-Optical Properties for Optical Imaging and Cancer Therapy. Acs Nano. 2017; 11: 6102-13.

147. Yu Q, Wang Y, Mei R, Yin Y, You J, Chen L. Polystyrene Encapsulated SERS Tags as Promising Standard Tools: Simple and Universal in Synthesis; Highly Sensitive and Ultrastable for Bioimaging. Analytical Chemistry. 2019; 91: 5270-7.

148. Zhai WY, He CL, Wu L, Zhou Y, Chen HR, Chang J, et al. Degradation of hollow mesoporous silica nanoparticles in human umbilical vein endothelial cells. Journal of Biomedical Materials Research Part B-Applied Biomaterials. 2012; 100B: 1397-403.

149. Hanske C, Sanz-Ortiz MN, Liz-Marzan LM. Silica-Coated Plasmonic Metal Nanoparticles in Action. Advanced Materials. 2018; 30

150. Stober W, Fink A, Bohn E. CONTROLLED GROWTH OF MONODISPERSE SILICA SPHERES IN MICRON SIZE RANGE. Journal of Colloid and Interface Science. 1968; 26: 62-\&.

151. Liz-Marzan LM, Giersig M, Mulvaney P. Synthesis of nanosized gold-silica core-shell particles. Langmuir. 1996; 12: 4329-35.

152. Doering WE, Nie SM. Spectroscopic tags using dye-embedded nanoparticles and surface-enhanced Raman scattering. Analytical Chemistry. 2003; 75: 6171-6.

153. Mulvaney SP, Musick MD, Keating CD, Natan MJ. Glass-coated, analyte-tagged nanoparticles: A new tagging system based on detection with surface-enhanced Raman scattering. Langmuir. 2003; 19: 4784-90.

154. Shah KW, Sreethawong T, Liu S-H, Zhang S-Y, Tan LS, Han M-Y. Aqueous route to facile, efficient and functional silica coating of metal nanoparticles at room temperature. Nanoscale. 2014; 6: 11273-81.

155. Graf C, Vossen DLJ, Imhof A, van Blaaderen A. A general method to coat colloidal particles with silica. Langmuir. 2003; 19: 6693-700.

156. Kuestner B, Gellner M, Schuetz M, Schoeppler F, Marx A, Stroebel P, et al. SERS Labels for Red Laser Excitation: Silica-Encapsulated SAMs on Tunable Gold/Silver Nanoshells. Angewandte Chemie-International Edition. 2009; 48: 1950-3.

157. Schuetz M, Kuestner B, Bauer M, Schmuck C, Schluecker S. Synthesis of Glass-Coated SERS Nanoparticle Probes via SAMs with Terminal SiO2 Precursors. Small. 2010; 6: 733-7.

158. Mir-Simon B, Reche-Perez I, Guerrini L, Pazos-Perez N, Alvarez-Puebla RA. Universal One-Pot and Scalable Synthesis of SERS Encoded Nanoparticles. Chemistry of Materials. 2015; 27: 950-8.

159. Fales AM, Yuan H, Vo-Dinh T. Silica-coated gold nanostars for combined surface-enhanced Raman scattering (SERS) detection and singlet-oxygen generation: a potential nanoplatform for theranostics. Langmuir. 2011; 27: 12186-90.

160. Montano-Priede JL, Coelho JP, Guerrero-Martinez A, Pena-Rodriguez O, Pal U. Fabrication of Monodispersed Au@SiO2 Nanoparticles with Highly Stable Silica Layers by Ultrasound-Assisted Stober Method. Journal of Physical Chemistry C. 2017; 121: 9543-51.

161. Monopoli MP, Aberg C, Salvati A, Dawson KA. Biomolecular coronas provide the biological identity of nanosized materials. Nature Nanotechnology. 2012; 7: 779-86.
162. Nel AE, Maedler L, Velegol D, Xia T, Hoek EMV, Somasundaran P, et al. Understanding biophysicochemical interactions at the nano-bio interface. Nature Materials. 2009; 8: 543-57.

163. Chithrani BD, Chan WCW. Elucidating the mechanism of cellular uptake and removal of protein-coated gold nanoparticles of different sizes and shapes. Nano Letters. 2007; 7: 1542-50.

164. Zheng X-S, Hu P, Cui Y, Zong C, Feng J-M, Wang X, et al. BSA-Coated Nanoparticles for Improved SERS-Based Intracellular $\mathrm{pH}$ Sensing. Analytical Chemistry. 2014; 86: 12250-7.

165. Niu X, Chen H, Wang Y, Wang W, Sun X, Chen L. Upconversion Fluorescence-SERS Dual-Mode Tags for Cellular and in Vivo Imaging. Acs Applied Materials \& Interfaces. 2014; 6: 5152-60.

166. Blanco-Covian L, Montes-Garcia V, Girard A, Teresa Fernandez-Abedul M, Perez-Juste J, Pastoriza-Santos I, et al. Au@Ag SERRS tags coupled to a lateral flow immunoassay for the sensitive detection of pneumolysin. Nanoscale. 2017; 9: 2051-8.

167. Wang Z, Zong S, Wang Y, Li N, Li L, Lu J, et al. Screening and multiple detection of cancer exosomes using an SERS-based method. Nanoscale. 2018; 10: 9053-62.

168. Yang K, Hu Y, Dong N, Zhu G, Zhu T, Jiang N. A novel SERS-based magnetic aptasensor for prostate specific antigen assay with high sensitivity. Biosensors \& Bioelectronics. 2017; 94: 286-91.

169. Qi L, Xiao M, Wang F, Wang L, Ji W, Man $\mathrm{T}$, et al. Poly-cytosine-mediated nanotags for SERS detection of $\mathrm{Hg} 2+$. Nanoscale. 2017; 9: 14184-91.

170. Ye S, Li X, Wang M, Tang B. Fluorescence and SERS Imaging for the Simultaneous Absolute Quantification of Multiple miRNAs in Living Cells. Analytical Chemistry. 2017; 89: 5124-30.

171. Simoncelli S, Roller E-M, Urban P, Schreiber R, Turberfield AJ, Liedl T, et al. Quantitative Single-Molecule Surface Enhanced Raman Scattering by Optothermal Tuning of DNA Origami-Assembled Plasmonic Nanoantennas. Acs Nano. 2016; 10: 9809-15.

172. Tam NCM, Scott BMT, Voicu D, Wilson BC, Zheng G. Facile Synthesis of Raman Active Phospholipid Gold Nanoparticles. Bioconjugate Chemistry. 2010; 21: 2178-82.

173. Ip S, MacLaughlin CM, Gunari N, Walker GC. Phospholipid Membrane Encapsulation of Nanoparticles for Surface-Enhanced Raman Scattering. Langmuir. 2011; 27: 7024-33.

174. Stewart AF, Lee A, Ahmed A, Ip S, Kumacheva E, Walker GC. Rational Design for the Controlled Aggregation of Gold Nanorods via Phospholipid Encapsulation for Enhanced Raman Scattering. Acs Nano. 2014; 8: 5462-7.

175. Su X, Wang Y, Wang W, Sun K, Chen L. Phospholipid Encapsulated AuNR@Ag/Au Nanosphere SERS Tags with Environmental Stimulus Responsive Signal Property. Acs Applied Materials \& Interfaces. 2016; 8: 10201-11.

176. Tam NCM, McVeigh PZ, MacDonald TD, Farhadi A, Wilson BC, Zheng G. Porphyrin-Lipid Stabilized Gold Nanoparticles for Surface Enhanced Raman Scattering Based Imaging. Bioconjugate Chemistry. 2012; 23: 1726-30.

177. Lee J-H, Shin Y, Lee W, Whang K, Kim D, Lee LP, et al. General and programmable synthesis of hybrid liposome/metal nanoparticles. Science Advances. 2016; 2.

178. Zavaleta CL, Smith BR, Walton I, Doering W, Davis G, Shojaei B, et al. Multiplexed imaging of surface enhanced Raman scattering nanotags in living mice using noninvasive Raman spectroscopy. Proc Natl Acad Sci U S A. 2009; 106: 13511-6.

179. Maeda H. Toward a full understanding of the EPR effect in primary and metastatic tumors as well as issues related to its heterogeneity. Advanced Drug Delivery Reviews. 2015; 91: 3-6.

180. Kobayashi H, Watanabe R, Choyke PL. Improving Conventional Enhanced Permeability and Retention (EPR) Effects; What Is the Appropriate Target? Theranostics. 2014; 4: 81-9.

181. Fang J, Nakamura H, Maeda H. The EPR effect: Unique features of tumor blood vessels for drug delivery, factors involved, and limitations and augmentation of the effect. Advanced Drug Delivery Reviews. 2011; 63: $136-51$.

182. Nayak TR, Andreou C, Oseledchyk A, Marcus WD, Wong HC, Massague J, et al. Tissue factor-specific ultra-bright SERRS nanostars for Raman detection of pulmonary micrometastases. Nanoscale. 2017; 9: $1110-9$.

183. Ou YC, Webb JA, O'Brien CM, Pence IJ, Lin EC, Paul EP, et al. Diagnosis of immunomarkers in vivo via multiplexed surface enhanced Raman spectroscopy with gold nanostars. Nanoscale. 2018; 10: 13092-105.

184. Ou YC, Wen X, Johnson CA, Shae D, Ayala OD, Webb JA, et al. Multimodal Multiplexed Immunoimaging with Nanostars to Detect Multiple Immunomarkers and Monitor Response to Immunotherapies. ACS Nano. 2020; 14: 651-63. 
185. Wang J, Liang D, Feng J, Tang X. Multicolor Cocktail for Breast Cancer Multiplex Phenotype Targeting and Diagnosis Using Bioorthogonal Surface-Enhanced Raman Scattering Nanoprobes. Anal Chem. 2019; 91: 11045-54.

186. de Albuquerque CDL, Schultz ZD. Super-resolution Surface-Enhanced Raman Scattering Imaging of Single Particles in Cells. Anal Chem. 2020; 92: 9389-98.

187. Li M, Banerjee SR, Zheng C, Pomper MG, Barman I. Ultrahigh affinity Raman probe for targeted live cell imaging of prostate cancer. Chem Sci. 2016; 7: 6779-85.

188. Zhang Y, Zhou W, Xue Y, Yang J, Liu D. Multiplexed Imaging of Trace Residues in a Single Latent Fingerprint. Anal Chem. 2016; 88: 12502-7.

189. Zhang D, Huang L, Liu B, Ni H, Sun L, Su E, et al. Quantitative and ultrasensitive detection of multiplex cardiac biomarkers in lateral flow assay with core-shell SERS nanotags. Biosens Bioelectron. 2018; 106: 204-11.

190. Sanchez-Purra M, Roig-Solvas B, Versiani A, Rodriguez-Quijada C, de Puig H, Bosch I, et al. Design of SERS nanotags for multiplexed lateral flow immunoassays. Mol Syst Des Eng. 2017; 2: 401-9.

191. Du Z, Qi Y, He J, Zhong D, Zhou M. Recent advances in applications of nanoparticles in SERS in vivo imaging. Wiley Interdiscip Rev Nanomed Nanobiotechnol. 2021; 13: e1672.

192. Li Y, Wei Q, Ma F, Li X, Liu F, Zhou M. Surface-enhanced Raman nanoparticles for tumor theranostics applications. Acta Pharm Sin B. 2018; 8: 349-59.

193. Nicolson F, Kircher MF, Stone N, Matousek P. Spatially offset Raman spectroscopy for biomedical applications. Chem Soc Rev. 2021; 50: 556-68.

194. Kircher MF, De La Zerda A, Jokerst JV, Zavaleta CL, Kempen PJ, Mittra E, et al. A brain tumor molecular imaging strategy using a new triple-modality MRI-photoacoustic-Raman nanoparticle. Nature Medicine. 2012; 18: 829-34.

195. Shaffer TM, Wall MA, Harmsen S, Longo VA, Drain CM, Kircher MF, et al. Silica nanoparticles as substrates for chelator-free labeling of oxophilic radioisotopes. Nano Lett. 2015; 15: 864-8.

196. Shaffer TM, Harmsen S, Khwaja E, Kircher MF, Drain CM, Grimm J. Stable Radiolabeling of Sulfur-Functionalized Silica Nanoparticles with Copper-64. Nano Lett. 2016; 16: 5601-4.

197. Dong YC, Hajfathalian M, Maidment PSN, Hsu JC, Naha PC, Si-Mohamed S, et al. Effect of Gold Nanoparticle Size on Their Properties as Contrast Agents for Computed Tomography. Sci Rep. 2019; 9: 14912.

198. Liu Y, Ashton JR, Moding EJ, Yuan H, Register JK, Fales AM, et al. A Plasmonic Gold Nanostar Theranostic Probe for In Vivo Tumor Imaging and Photothermal Therapy. Theranostics. 2015; 5: 946-60.

199. Gao Y, Li Y, Chen J, Zhu S, Liu X, Zhou L, et al. Multifunctional gold nanostar-based nanocomposite: Synthesis and application for noninvasive MR-SERS imaging-guided photothermal ablation. Biomaterials. 2015; 60: 31-41.

200. Zhang Y, Liu Z, Thackray BD, Bao Z, Yin X, Shi F, et al. Intraoperative Raman-Guided Chemo-Photothermal Synergistic Therapy of Advanced Disseminated Ovarian Cancers. Small. 2018; 14.

201. He J, Dong J, Hu Y, Li G, Hu Y. Design of Raman tag-bridged core-shell Au@Cu-3(BTC)(2) nanoparticles for Raman imaging and synergistic chemo-photothermal therapy. Nanoscale. 2019; 11: 6089-100.

202. He J, Qiao Y, Zhang H, Zhao J, Li W, Xie T, et al. Gold-silver nanoshells promote wound healing from drug-resistant bacteria infection and enable monitoring via surface-enhanced Raman scattering imaging. Biomaterials. 2020; 234: 119763.

203. He J, Wei Q, Wang S, Hua S, Zhou M. Bioinspired protein corona strategy enhanced biocompatibility of Ag-Hybrid hollow Au nanoshells for surface-enhanced Raman scattering imaging and on-demand activation tumor-phototherapy. Biomaterials. 2021; 271: 120734.

204. Garai E, Sensarn S, Zavaleta CL, Van de Sompel D, Loewke NO, Mandella MJ, et al. High-sensitivity, real-time, ratiometric imaging of surface-enhanced Raman scattering nanoparticles with a clinically translatable Raman endoscope device. J Biomed Opt. 2013; 18: 096008.

205. Bohndiek SE, Wagadarikar A, Zavaleta CL, Van de Sompel D, Garai E, Jokerst JV, et al. A small animal Raman instrument for rapid, wide-area, spectroscopic imaging. Proc Natl Acad Sci U S A. 2013; 110: 12408-13. 